IFUM-919-FT

\title{
Anomalous dimension with wrapping at four loops in $\mathcal{N}=4 \mathrm{SYM}$
}

\author{
F. Fiamberti ${ }^{a, b}$, A. Santambrogio ${ }^{b}$, C. Sieg ${ }^{b}$, D. Zanon ${ }^{a, b} *$ \\ a Dipartimento di Fisica, Università degli Studi di Milano, \\ Via Celoria 16, 20133 Milano, Italy \\ ${ }^{b}$ INFN-Sezione di Milano, \\ Via Celoria 16, 20133 Milano, Italy
}

\begin{abstract}
In this paper we give all the details of the calculation that we presented in our previous paper arXiv:0712.3522, concerning the four-loop anomalous dimension of the Konishi descendant $\operatorname{tr}(\phi Z \phi Z-\phi \phi Z Z)$ in the $S U(2)$ sector of the $\mathcal{N}=4$ planar SYM theory. We explicitly consider all the wrapping diagrams that we compute using an $\mathcal{N}=1$ superspace approach and Gegenbauer polynomial $x$-space techniques.
\end{abstract}

*francesco.fiamberti@mi.infn.it

alberto.santambrogio@mi.infn.it

csieg@mi.infn.it

daniela.zanon@mi.infn.it 


\section{Introduction}

Recently we have presented [1] the calculation of the anomalous dimension of the composite operator $\operatorname{tr}(\phi Z \phi Z-\phi \phi Z Z)$ at four loops in the $\mathcal{N}=4$ planar supersymmetric Yang-Mills theory, where $\phi$ and $Z$ are two complex scalar fields of the theory.

The importance of this calculation resides in the fact that this is the simplest case in which the so-called wrapping effects are present: at a given perturbative order $K$ in

$$
\lambda=\frac{g^{2} N}{16 \pi^{2}},
$$

where $g=g_{\mathrm{YM}}$ is the Yang-Mills coupling constant, the range of the interactions between adjacent fields grows with the perturbative order as $K+1$. For an operator with $L$ fields we should expect new effects when the range exceeds $L$, that is at order $g^{2 L}$. It turns out that $L=4$ is the simplest case in which these effects can be seen (operators with $L=2$ and $L=3$ are automatically BPS).

Understanding these wrapping effects is a crucial step towards a comparison between the spectrum of the anomalous dimensions of gauge invariant operators of planar $\mathcal{N}=4$ SYM and the spectrum of strings on $\mathrm{AdS}_{5} \times \mathrm{S}^{5}$, as predicted by the AdS/CFT conjecture [2].

Indeed, in the case of long operators, the comparison between the spectra of the two theories is now at hand, thanks to the recent progress in understanding their integrability properties. On the gauge theory side it was crucial the realization $[3,4]$ that the planar one-loop dilatation operator of $\mathcal{N}=4$ SYM maps into the Hamiltonian of an integrable spin chain. This spin chain picture was extended to higher orders in perturbation theory $[5,6]$ and thanks to the integrability properties of the system the computation of the anomalous dimensions was then reduced to finding solutions of the associated Bethe equations [7]. This program cannot be applied to short operators since the Bethe ansatz is only asymptotic and wrapping effects appear at order $g^{2 L}$ : the interaction is no longer localized in some limited region along the state and asymptotic states cannot be defined.

Clearly from the perspective of integrable models including wrapping effects is very important and several papers have addressed this issue. In [8] the properties of wrapping interactions have been analyzed in terms of Feynman diagrams. In [9] wrapping interactions in the BMN matrix model have been discussed. In $[10,11]$ it was proposed to study wrapping interactions via the thermodynamic Bethe ansatz, while in [12] it was assumed that wrapping effects might be described by the Hubbard model. In [13] the form of wrapping contributions at four loops was conjectured by using restrictions from the BFKL equation [14-16]. In [17] wrapping effects in some simple spin chain models 
were studied. On the string theory side, finite size contributions have been analyzed in several recent papers [18-25].

In order to test these proposals it is crucial to perform an explicit field theoretical computation of an anomalous dimension at an order in which wrapping contributions become relevant. As mentioned above the simplest such a case is the four-loop anomalous dimension of the Konishi descendant $\operatorname{tr}(\phi Z \phi Z-\phi \phi Z Z)$ in the $S U(2)$ sector of the theory.

In this paper we describe in detail the computation we have presented in [1], with the aim of supplying the interested reader with all the details needed to reproduce the calculation.

The complete four-loop Feynman graph calculation is very complicated. However one can take advantage of the approach based on the asymptotic dilatation operator and avoid the explicit computation of some of the most difficult classes of graphs. Indeed the contribution from all non-wrapping graphs, corresponding to interactions of range from one to four, is included in the four-loop asymptotic dilatation operator together with the interactions of range five.

Then one is left with two operations to be done: the subtraction of the rangefive contributions from the dilatation operator, and the computation of the wrapping graphs. These two operations are drastically simplified by means of $\mathcal{N}=1$ superspace techniques. The integrals that we produce after $D$-algebra manipulations on the supergraphs are computed by the Gegenbauer polynomial $x$-space technique (GPXT) [26] and by the method of uniqueness [27].

The anomalous dimension of a composite operator $\mathcal{O}$ is extracted from the $\frac{1}{\epsilon}$ pole of the graphs contributing to its renormalization in a standard way. In the special case in which the operator is multiplicatively renormalized the anomalous dimension is given by

$$
\gamma(\mathcal{O})=\lim _{\varepsilon \rightarrow 0}\left[\varepsilon g \frac{\mathrm{d}}{\mathrm{d} g} \log \mathcal{Z}_{\mathcal{O}}(g, \varepsilon)\right]
$$

where

$$
\mathcal{O}_{\text {ren }}=\mathcal{Z}_{\mathcal{O}} \mathcal{O}_{\text {bare }}
$$

In the general case of operator mixing, the last equation should be rewritten in matrix form and the anomalous dimensions are given by the eigenvalues of the matrix appearing on the right hand side of (1.2). In our case we have two mixing operators but it turns out that the combination $\operatorname{tr}(\phi Z \phi Z-\phi \phi Z Z)$ is an exact eigenvector of the renormalization matrix to all orders, so it is multiplicatively renormalized.

The most remarkable outcome of our calculation is that at four-loop order wrapping interactions give rise to contributions proportional to $\zeta(5)$ increasing the level of transcendentality of the anomalous dimensions. Our result rules out the previous conjectures 
on how to treat wrapping contributions proposed in $[12,13,28]$.

Another computation of the same anomalous dimension appeared later on in [29]. The results presented there do not match our findings, but the authors did not attempt a comparison with the computations we had reported. The result of [29] contains a trivial error in the final step, however even after correcting it, the result is different from ours. Finding the origin of the discrepancy is not an easy task. The authors of [29] used exactly the same strategy as ours but their calculation of the wrapping diagrams is performed using a component field approach. Thus the number of diagrams they have to compute is huge, supersymmetry is not maintained explicitly, renormalization is subtle and partial comparison with our classes of diagrams is almost impossible. Needless to say that keeping under control such a large number of contributions is very difficult. On the contrary using superfields the calculation is much simpler since several cancellations are automatic, and the number of relevant diagrams is significantly reduced. In any event we hope that all the detailed explanations we are going to present in this paper will help to clarify the situation.

Our paper is organized as follows. In Section 2 we recall the basic ingredients of the $\mathcal{N}=1$ superspace approach, and we discuss an important result on the displacement of spinorial derivatives on a supergraph that allows us to drastically reduce the number of potential contributions. In Section 3 we describe how to subtract the range-five terms from the asymptotic Hamiltonian and we build the effective Hamiltonian containing all the contributions from range one up to range four. Thanks to a cancellation described in Appendix A, the range-five subtraction procedure can be performed without computing any Feynman diagram. However in order to provide a further check of our procedure we have computed all range-five graphs explicitly. This is reported in Appendix B. Subsection 4.1 contains the calculation of the wrapping supergraphs. The full list of them is reported in Appendix C, with a description of all the cancellations occurring among them and with all the integrals produced after completion of the $D$-algebra. The GPXT method used to compute the momentum integrals is summarized in Subsection 4.2 and Appendix D. Using the results obtained so far in Subsection 4.3 we are able to give an expression for the four-loop wrapping dilatation operator. In Section 5 we collect our results and we give the final answer for the Konishi anomalous dimension at four loops.

\section{$2 \mathcal{N}=1$ superspace approach}

We are going to describe the calculation of the anomalous dimension of the composite operator $\operatorname{tr}(\phi Z \phi Z-\phi \phi Z Z)$ at four loops in the planar limit. In order to streamline the 
calculation we decided to work using a $\mathcal{N}=1$ superspace formalism. In this section we review the most relevant features of this approach needed to perform our calculation.

The action of $\mathcal{N}=4 \mathrm{SYM}$ can be written using $\mathcal{N}=1$ superspace in terms of one real vector superfield $V$ and three chiral superfields $\phi^{i}$ (we follow the notations and conventions of [30])

$$
\begin{aligned}
S= & \int \mathrm{d}^{4} x \mathrm{~d}^{4} \theta \operatorname{tr}\left(\mathrm{e}^{-g V} \bar{\phi}_{i} \mathrm{e}^{g V} \phi^{i}\right)+\frac{1}{2 g^{2}} \int \mathrm{d}^{4} x \mathrm{~d}^{2} \theta \operatorname{tr}\left(W^{\alpha} W_{\alpha}\right) \\
& +i \frac{g}{3 !} \int \mathrm{d}^{4} x \mathrm{~d}^{2} \theta \epsilon^{i j k} \operatorname{tr}\left(\phi_{i}\left[\phi_{j}, \phi_{k}\right]\right)+\text { h.c. },
\end{aligned}
$$

where $W_{\alpha}=i \bar{D}^{2}\left(\mathrm{e}^{-g V} D_{\alpha} \mathrm{e}^{g V}\right)$, and $V=V^{a} T^{a}, \phi^{i}=\phi_{i}^{a} T^{a}, i=1,2,3, T^{a}$ being matrices satisfying the $S U(N)$ algebra

$$
\left[T_{a}, T_{b}\right]=i f_{a b c} T_{c}
$$

and being normalized as

$$
\operatorname{tr}\left(T_{a} T_{b}\right)=\delta_{a b}
$$

We will usually denote the three chiral superfields $\phi^{i}$ as $(\phi, \psi, Z)$.

We want to study the renormalization of the composite chiral operators

$$
\mathcal{O}_{1}=\operatorname{tr}(\phi Z \phi Z), \quad \mathcal{O}_{2}=\operatorname{tr}(\phi \phi Z Z)
$$

which mix under renormalization. To this end, the first step is the construction of all the supergraphs containing one operator insertion. The Feynman rules can be derived from the action (2.1). In momentum space we have the superfield propagators

$$
\left\langle V^{a} V^{b}\right\rangle=-\frac{\delta^{a b}}{p^{2}}, \quad\left\langle\phi_{i}^{a} \bar{\phi}_{j}^{b}\right\rangle=\delta_{i j} \frac{\delta^{a b}}{p^{2}},
$$

while the vertices are read-off directly from the interaction terms in (2.1), with additional $\bar{D}^{2}, D^{2}$ factors for chiral, antichiral lines respectively. The ones that we need are the following

$$
\begin{array}{rlrl}
V_{1} & =i g f_{a b c} \delta^{i j} \bar{\phi}_{i}^{a} V^{b} \phi_{j}^{c}, & V_{2} & =\frac{g^{2}}{2} \delta^{i j} f_{a d m} f_{b c m} V^{a} V^{b} \bar{\phi}_{i}^{c} \phi_{j}^{d}, \\
V_{3} & =-\frac{g}{3 !} \epsilon^{i j k} f_{a b c} \phi_{i}^{a} \phi_{j}^{b} \phi_{k}^{c}, & \bar{V}_{3}=-\frac{g}{3 !} \epsilon^{i j k} f_{a b c} \bar{\phi}_{i}^{a} \bar{\phi}_{j}^{b} \bar{\phi}_{k}^{c} .
\end{array}
$$

Vertices containing three or more vector superfields $V$ do not enter our calculation.

The general idea is to consider a given supergraph and to perform the $D$-algebra in order to reduce it to a standard graph. In doing so one usually produces a large number 
of contributions, but most of them are not relevant for the computation of the anomalous dimension of the composite operator since they give rise to finite integrals. Thus it is very useful to know and to exploit $D$-algebra manipulations that can be made on the supergraph and that are legal only up to finite terms. In the next subsection we are going to describe a property of the supergraphs that was very important in computing our diagrams.

\subsection{A useful proof}

We consider diagrams with one operator insertion, built using only chiral, antichiral, single-vector and double-vector vertices. We are interested in isolating those contributions which give rise to divergent integrals, i.e. the ones which will contribute to the anomalous dimension. Here we want to prove that in order to produce divergent contributions we have to perform and complete the $D$-algebra in such a way that no spinor derivative is moved out of the diagram onto the external lines, except for derivatives on scalar propagators that do not belong to any loop from the start. Let us define the following quantities:

- $V_{C}=$ number of chiral vertices

- $V_{A}=$ number of antichiral vertices

- $V_{V}^{(1)}=$ number of single-vector vertices

- $V_{V}^{(2)}=$ number of double-vector vertices

- $p_{C}=$ number of scalar propagators belonging to at least one loop

- $p_{V}=$ number of vector propagators

- $p=$ total number of propagators belonging to at least one loop

- $E=$ number of scalar propagators not belonging to any loop.

- $N_{\ell}=$ number of loops

- $N_{D}, N_{\bar{D}}$ : number of spinor derivatives

In all our diagrams we have $V_{A}=V_{C}$ and $N_{D}=N_{\bar{D}}$, and $E$ is equal to the number of chiral vertices not belonging to any loop.

Moreover the number of outgoing external lines equals the number of fields in the composite operator. From each chiral or antichiral vertex three scalar lines start. From each single-vector vertex, two scalar and one vector propagators start. From each doublevector vertex, two scalar and two vector lines start. 
Given these relations, we can write

$$
p_{C}=\frac{1}{2}\left[3\left(V_{C}+V_{A}\right)+2\left(V_{V}^{(1)}+V_{V}^{(2)}\right)\right]-E, \quad p_{V}=\frac{1}{2}\left[V_{V}^{(1)}+2 V_{V}^{(2)}\right] .
$$

At each chiral vertex, two out of the three scalar lines have a $\bar{D}^{2}$.

At each antichiral vertex, two out of the three scalar lines have a $D^{2}$.

At each single-vector or double-vector vertex, one of the scalar lines has a $\bar{D}^{2}$, while the other one has a $D^{2}$. So we have

$$
N_{D}=4 V_{C}+2\left(V_{V}^{(1)}+V_{V}^{(2)}\right), \quad N_{\bar{D}}=4 V_{A}+2\left(V_{V}^{(1)}+V_{V}^{(2)}\right) .
$$

Then we can write

$$
p=p_{C}+p_{V}=\frac{1}{2}\left[V_{C}+V_{A}+N_{D}+V_{V}^{(1)}+2 V_{V}^{(2)}\right]-E .
$$

In order to obtain a logarithmic divergent diagram, the $D$-algebra must produce a number of standard derivatives equal to

$$
2 p-4 N_{\ell}
$$

We need a $D$ (and a $\bar{D}$ ) to create each standard derivative. Furthermore, we need $2 N_{\ell}$ extra $D$ 's (and $\bar{D}$ 's) to complete the superspace $\mathrm{d}^{4} \theta$ integration for the $N_{\ell}$ loops. So after performing the $D$-algebra, a momentum integral with a surface divergence can be found only if at least

$$
2 p-2 N_{\ell}
$$

out of all the $D$ 's are not brought outside the diagram on the external lines.

A $D^{2}$ can always be moved onto each scalar propagator which is not part of a loop: either it is already there at the beginning of the $D$-algebra, or it can be moved there through an integration by parts at the internal vertex at which the external propagator is linked. So the effective number of $D$ 's available in the loops is

$$
N_{D}-2 E
$$

During the $D$-algebra manipulations we will be allowed to move some $D$ 's outside the diagram only if their number at the start exceeds the number we need in order to produce a divergent integral. So we should have

$$
N_{D}-2 E>2 p-2 N_{\ell}=\left[V_{C}+V_{A}+N_{D}+V_{V}^{(1)}+2 V_{V}^{(2)}\right]-2 E-2 N_{\ell},
$$


that is

$$
N_{\ell}>\frac{1}{2}\left[V_{C}+V_{A}+V_{V}^{(1)}+2 V_{V}^{(2)}\right]
$$

This condition is never satisfied by the diagrams in the class we are studying, as can be seen using Euler's formula for connected graphs

$$
V-P+F=2
$$

where $V$ denotes the number of vertices, $P$ is the number of propagators and $F$ is the number of faces.

In our case (remember that the composite operator behaves as an additional vertex) we have

$$
V=V_{A}+V_{C}+V_{V}^{(1)}+V_{V}^{(2)}+1, \quad P=p+E, \quad F=N_{\ell}+1,
$$

which leads to

$$
N_{\ell}=\frac{1}{2}\left[V_{C}+V_{A}+V_{V}^{(1)}+2 V_{V}^{(2)}\right] .
$$

We conclude that in order to produce a divergent contribution no spinor derivatives can be brought outside the diagram.

All our $D$-algebra manipulations were performed making use of this property: only the contributions in which all spinor derivatives stay in the loops were kept. Clearly this rule allowed to discard a huge number of irrelevant contributions.

\section{Subtraction of range-five diagrams from the asymp- totic Hamiltonian}

In this section we are going to describe how to compute the contributions from the diagrams with range from one to four with no wrapping interactions. In order to avoid their explicit, very complicated Feynman graph computation we take advantage of the asymptotic four-loop dilatation operator $D_{4}$, whose expression was given in the $S U(2)$ sector by [31]: 


$$
\begin{aligned}
D_{4}= & -(560+4 \beta)\{\} \\
& +\left(1072+12 \beta+8 \epsilon_{3 a}\right)\{1\} \\
& -\left(84+6 \beta+4 \epsilon_{3 a}\right)\{1,3\}-4\{1,4\}-\left(302+4 \beta+8 \epsilon_{3 a}\right)(\{1,2\}+\{2,1\}) \\
& +\left(4 \beta+4 \epsilon_{3 a}+2 i \epsilon_{3 c}-4 i \epsilon_{3 d}\right)\{1,3,2\}+\left(4 \beta+4 \epsilon_{3 a}-2 i \epsilon_{3 c}+4 i \epsilon_{3 d}\right)\{2,1,3\} \\
& +\left(4-2 i \epsilon_{3 c}\right)(\{1,2,4\}+\{1,4,3\})+\left(4+2 i \epsilon_{3 c}\right)(\{1,3,4\}+\{2,1,4\}) \\
& +\left(96+4 \epsilon_{3 a}\right)(\{1,2,3\}+\{3,2,1\}) \\
& -\left(12+2 \beta+4 \epsilon_{3 a}\right)\{2,1,3,2\}+\left(18+4 \epsilon_{3 a}\right)(\{1,3,2,4\}+\{2,1,4,3\}) \\
& -\left(8+2 \epsilon_{3 a}+2 i \epsilon_{3 b}\right)(\{1,2,4,3\}+\{1,4,3,2\}) \\
& -\left(8+2 \epsilon_{3 a}-2 i \epsilon_{3 b}\right)(\{2,1,3,4\}+\{3,2,1,4\}) \\
& -10(\{1,2,3,4\}+\{4,3,2,1\}),
\end{aligned}
$$

where $\epsilon_{3 a}, \epsilon_{3 b}, \epsilon_{3 c}$ and $\epsilon_{3 d}$ parameterize the invariance of the Hamiltonian under similarity transformations. In a perturbative approach their values depend on the choice of the renormalization scheme. The parameter $\beta=4 \zeta(3)$ comes from the dressing phase $[28,31-34]$.

The permutation structures appearing in (3.1) are defined as

$$
\left\{a_{1}, \ldots, a_{n}\right\}=\sum_{r=0}^{L-1} \mathrm{P}_{a_{1}+r a_{1}+r+1} \cdots \mathrm{P}_{a_{n}+r a_{n}+r+1}
$$

where $\mathrm{P}_{a a+1}$, with the cyclic identification $\mathrm{P}_{a a+1} \simeq \mathrm{P}_{a+L a+L+1}$, permutes the flavours of the $a$ th and $(a+1)$ th site when acting on a cyclic state of length $L$. The number of nearest neighbours, interacting in the flavour space, is extracted from the list of integers $a_{1}, \ldots, a_{n}$ in the permutation structure as

$$
\kappa=2+\max _{a_{1} \ldots a_{n}}-\min _{a_{1} \ldots a_{n}}
$$

It is limited from above by the order $K$ in the perturbation expansion in powers $\lambda^{K}$ as $\kappa \leq K+1$. The asymptotic regime in which $D_{4}$ can be trustfully applied is determined by the relation $L>K$. Some rules valid in the asymptotic case for the manipulation of these structures can be found in [35].

The expression (3.1) contains contributions which describe the permutations among $\kappa=5$ neighbouring legs. Therefore it is correct only if applied to a state in the asymptotic sense, i.e. the number of sites in the state has to be five or more. We can correct 
it for the application on a length four state: the contributions from all Feynman diagrams which describe the interactions of five neighbouring legs have to be replaced by the contributions from all four-loop wrapping interactions.

Before doing this, it is advantageous to change the basis from the permutation structures (3.2) to a set of functions which are directly related to the chiral structures of the Feynman supergraphs. At four loops we are lead to define the following chiral functions

$$
\begin{aligned}
\chi(a, b, c, d)= & \{\}-4\{1\}+\{a, b\}+\{a, c\}+\{a, d\}+\{b, c\}+\{b, d\}+\{c, d\} \\
& -\{a, b, c\}-\{a, b, d\}-\{a, c, d\}-\{b, c, d\}+\{a, b, c, d\}, \\
\chi(a, b, c)= & -\{\}+3\{1\}-\{a, b\}-\{a, c\}-\{b, c\}+\{a, b, c\}, \\
\chi(a, b)= & \{\}-2\{1\}+\{a, b\}, \\
\chi(1)= & -\{\}+\{1\}, \\
\chi()= & \{\} .
\end{aligned}
$$

The relevance of these functions stems from the fact that any Feynman supergraph produces a permutation structure given by one of them. Moreover we call them chiral because their form depends only on the structure of the chiral interactions of the diagram, while they are completely insensitive to the presence of vector lines.

The form of these functions can be easily derived: $\chi()$ is just the identity, corresponding to a Feynman supergraph without chiral vertices. The first non-trivial function, $\chi(1)$ corresponds to the diagram given in Figure 1.

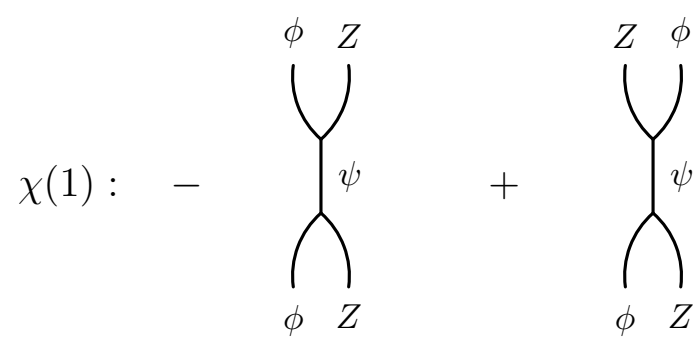

Figure 1: Building block

This diagram has two vertices $V_{3}$ and $\bar{V}_{3}$ (see (2.6) $)$ connected by a $\langle\psi \bar{\psi}\rangle$ superfield propagator, which is killed by the $D$-algebra. It is a sort of effective four-vertex, very similar to the one describing the scalar interaction in a component approach. This diagram is a "building block" for the construction of more complicated ones. 
Indeed it is easy to see that every four-loop supergraph contributing to the one-point function of a chiral operator in the $S U(2)$ sector, like our operators (2.4), are built by gluing together up to four of the building blocks given in Figure 1. The form of the chiral functions in (3.4) then follows consequently.

Let us notice that the number of arguments $n$, with $n=1, \ldots, 4$ at four loops, corresponds to the number of building blocks in our underlying Feynman graphs. The fact that the coefficients in each chiral structure sum up to zero guarantees that the two length $L$ BPS operators $\operatorname{tr}\left(Z^{L}\right)$ and $\operatorname{tr}\left(\phi Z^{L-1}\right)$ are protected.

In order to rewrite $D_{4}$ in (3.1) in terms of the chiral functions, we have to express the permutation structures (3.2) in terms of the chiral functions (3.4). We have

$$
\begin{aligned}
\{a, b, c, d\}= & \chi(a, b, c, d)+\chi(a, b, c)+\chi(a, b, d)+\chi(a, c, d)+\chi(b, c, d) \\
& +\chi(a, b)+\chi(a, c)+\chi(a, d)+\chi(b, c)+\chi(b, d)+\chi(c, d) \\
& +4 \chi(1)+\chi() \\
\{a, b, c\}= & \chi(a, b, c)+\chi(a, b)+\chi(a, c)+\chi(b, c)+3 \chi(1)+\chi(), \\
\{a, b\}= & \chi(a, b)+2 \chi(1)+\chi(), \\
\{1\}= & \chi(1)+\chi(), \\
\{\}= & \chi() .
\end{aligned}
$$

Making use of these relations we reexpress the four-loop dilatation operator (3.1) in terms of the chiral functions and obtain

$$
\begin{aligned}
D_{4}= & +200 \chi(1)-150[\chi(1,2)+\chi(2,1)]+8\left(10+\epsilon_{3 a}\right) \chi(1,3)-4 \chi(1,4) \\
& +60[\chi(1,2,3)+\chi(3,2,1)] \\
& +\left(8+2 \beta+4 \epsilon_{3 a}-4 i \epsilon_{3 b}+2 i \epsilon_{3 c}-4 i \epsilon_{3 d}\right) \chi(1,3,2) \\
& +\left(8+2 \beta+4 \epsilon_{3 a}+4 i \epsilon_{3 b}-2 i \epsilon_{3 c}+4 i \epsilon_{3 d}\right) \chi(2,1,3) \\
& -\left(4+4 i \epsilon_{3 b}+2 i \epsilon_{3 c}\right)[\chi(1,2,4)+\chi(1,4,3)] \\
& -\left(4-4 i \epsilon_{3 b}-2 i \epsilon_{3 c}\right)[\chi(1,3,4)+\chi(2,1,4)] \\
& -\left(12+2 \beta+4 \epsilon_{3 a}\right) \chi(2,1,3,2) \\
& +\left(18+4 \epsilon_{3 a}\right)[\chi(1,3,2,4)+\chi(2,1,4,3)] \\
& -\left(8+2 \epsilon_{3 a}+2 i \epsilon_{3 b}\right)[\chi(1,2,4,3)+\chi(1,4,3,2)] \\
& -\left(8+2 \epsilon_{3 a}-2 i \epsilon_{3 b}\right)[\chi(2,1,3,4)+\chi(3,2,1,4)] \\
& -10[\chi(1,2,3,4)+\chi(4,3,2,1)] .
\end{aligned}
$$

This representation is particularly useful since the chiral functions $\chi$ and not the permutation structures are directly related to the underlying Feynman supergraph. However 
there is still an ambiguity: two supergraphs which differ only by the arrangement of the flavour-neutral vector superfield lines contribute to the coefficient in front of the same chiral function. Therefore the chiral functions do not allow in general to extract information about the range of the interaction of the underlying Feynman supergraph.

In particular range-five diagrams in which the first or fifth line is connected to the rest of the diagram only via vector line(s) appear with the same chiral function as the corresponding range-four diagram in which the corresponding first or fifth line is a noninteracting spectator. We call those range-five diagrams which have a chiral function which does not capture the range of their interaction non-maximal range-five diagrams. They also have to be subtracted from $D_{4}$ and in general one should compute explicitly all these Feynman graphs. Luckily it turns out that there is no need to do that. In Appendix A we show that the non-maximal range-five interactions do not contribute to $D_{4}$. This means that we can determine the subtraction directly from $D_{4}$. This operation becomes particularly simple using the representation of $D_{4}$ in terms of the chiral functions $\chi$. We only have to neglect all the terms with chiral functions $\chi\left(a_{1}, \ldots, a_{n}\right)$ which describe a range-five interaction, i.e. which fulfill $\max _{a_{1} \ldots a_{n}}-\min _{a_{1} \ldots a_{n}}=3$. Hence we have to subtract from (3.6)

$$
\begin{aligned}
\delta D_{4}= & -10[\chi(1,2,3,4)+\chi(4,3,2,1)]+\left(18+4 \epsilon_{3 a}\right)[\chi(1,3,2,4)+\chi(2,1,4,3)] \\
& -\left(8+2 \epsilon_{3 a}+2 i \epsilon_{3 b}\right)[\chi(1,2,4,3)+\chi(1,4,3,2)] \\
& -\left(8+2 \epsilon_{3 a}-2 i \epsilon_{3 b}\right)[\chi(2,1,3,4)+\chi(3,2,1,4)] \\
& -\left(4+4 i \epsilon_{3 b}+2 i \epsilon_{3 c}\right)[\chi(1,2,4)+\chi(1,4,3)] \\
& -\left(4-4 i \epsilon_{3 b}-2 i \epsilon_{3 c}\right)[\chi(1,3,4)+\chi(2,1,4)] \\
& -4 \chi(1,4) .
\end{aligned}
$$

We should stress that due to the decomposition in (3.4), the subtraction is not only a simple neglection of all range-five permutation structures as attempted in [9] for the BMN matrix model. One has to modify also the coefficients of the permutation structures of lower range in (3.1).

In order to have an independent check of the whole procedure, we have found the above constructed range-five contributions (3.7) also through an explicit Feynman graph computation. We give in Appendix B all the details of the calculation. As a byproduct we have found in this way explicit values for the unphysical coefficients $\epsilon_{3 a}, \epsilon_{3 b}, \epsilon_{3 c}$. In the MS-scheme we have used, they are given by

$$
\epsilon_{3 a}=-4, \quad \epsilon_{3 b}=-i \frac{4}{3}, \quad \epsilon_{3 c}=i \frac{4}{3} .
$$


Thanks to the absence of non-maximal range-five interactions the explicit Feynman graph computation was not necessary. It was performed just as a further check on the range-five part of $D_{4}$.

We emphasize that a straightforward modification of the proof in Appendix A leads to the cancellation of this type of contributions also at higher loop orders. This feature is quite interesting since it allows to determine the necessary subtractions in the general case of $D_{K}$ applied to a length $L=K$ operator, with no need of Feynman diagram computations.

Finally after subtraction of the range-five contributions the four-loop dilatation operator becomes

$$
\begin{aligned}
D_{4}^{\text {sub }} \equiv D_{4}-\delta D_{4}= & +200 \chi(1)-150[\chi(1,2)+\chi(2,1)]+8\left(10+\epsilon_{3 a}\right) \chi(1,3) \\
& +60[\chi(1,2,3)+\chi(3,2,1)] \\
& +\left(8+2 \beta+4 \epsilon_{3 a}-4 i \epsilon_{3 b}+2 i \epsilon_{3 c}-4 i \epsilon_{3 d}\right) \chi(1,3,2) \\
& +\left(8+2 \beta+4 \epsilon_{3 a}+4 i \epsilon_{3 b}-2 i \epsilon_{3 c}+4 i \epsilon_{3 d}\right) \chi(2,1,3) \\
& -\left(12+2 \beta+4 \epsilon_{3 a}\right) \chi(2,1,3,2)
\end{aligned}
$$

We can now apply the subtracted dilatation operator to the states with $L=4$ sites. In the $S U(2)$ subsector there exist two composite operators of length $L=4$ and with two 'impurities' which mix under renormalization. The corresponding states are given by

$$
\mathcal{O}_{1}=\operatorname{tr}(\phi Z \phi Z), \quad \mathcal{O}_{2}=\operatorname{tr}(\phi \phi Z Z)
$$

Defining a two-dimensional vector $\overrightarrow{\mathcal{O}}=\left(\mathcal{O}_{1}, \mathcal{O}_{2}\right)^{\mathrm{t}}$, the application of the chiral structures to $\overrightarrow{\mathcal{O}}$ is captured by the replacements

$$
\begin{gathered}
\chi(1) \rightarrow M, \quad \chi(1,2) \rightarrow-M, \quad \chi(1,3) \rightarrow-2 M, \quad \chi(2,1) \rightarrow-M, \\
\chi(1,2,3) \rightarrow M, \quad \chi(3,2,1) \rightarrow M, \quad \chi(2,1,3) \rightarrow 2 M, \quad \chi(1,3,2) \rightarrow 2 M, \\
\chi(2,1,3,2) \rightarrow-2 M
\end{gathered}
$$

where the common mixing matrix is given by

$$
M=\left(\begin{array}{cc}
-4 & 4 \\
2 & -2
\end{array}\right)
$$

The eigenvectors and eigenvalues of $M$ are given by

$$
\begin{aligned}
& \mathcal{O}_{1}^{\prime}=\mathcal{O}_{1}+2 \mathcal{O}_{2}=\frac{1}{2}(3 \operatorname{tr}(\phi\{Z, \phi\} Z)-\operatorname{tr}(\phi[Z, \phi] Z)), \quad M\left(\begin{array}{c}
\mathcal{O}_{1}^{\prime} \\
\mathcal{O}_{2}^{\prime}
\end{array}\right)=\left(\begin{array}{c}
0 \\
-6 \mathcal{O}_{2}^{\prime}
\end{array}\right) . \\
& \mathcal{O}_{2}^{\prime}=\mathcal{O}_{1}-\mathcal{O}_{2}=\operatorname{tr}(\phi[Z, \phi] Z),
\end{aligned}
$$


These are directly the eigenvectors of $D_{4}^{\text {sub }}$ in (3.9), since in the $L=4$ operator basis (3.10) it is proportional to $M$. With $\beta=4 \zeta(3)$ and $\epsilon_{3 a}$ from (3.8) it assumes the form

$$
D_{4}^{\text {sub }} \rightarrow 4(121+12 \zeta(3)) M .
$$

In the next section we move our attention to the calculation of wrapping diagrams.

\section{Contributions from wrapping diagrams}

In this section we are going to face the computation of the wrapping contributions. This is the part of the analysis that necessarily requires the explicit computation of Feynman supergraphs. The first step will be to build all wrapping supergraphs, and for this we rely on the results of [8], where a systematic Feynman-diagrammatic analysis of wrapping interactions was performed.

In the present paper we are using $\mathcal{N}=1$ superspace techniques, so we need to adapt that approach for the construction of all the wrapping supergraphs contributing to the renormalization of the chiral operators (3.10).

The second step will be to compute the four-loop momentum integrals using the GPXT.

Finally we collect these results in a wrapping four-loop dilatation operator.

\subsection{Wrapping supergraphs}

First of all we have a set of wrapping graphs with only chiral interactions, which are shown in Figure 2.
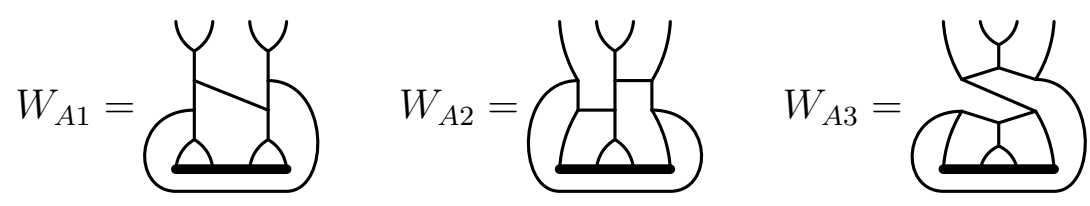

Figure 2: Wrapping diagrams with only chiral interactions

With the identification of the first and the fifth lines, we can still use the defini- 
tion (3.2) for the permutation operators. Then the chiral structures will be

$$
\begin{aligned}
& W_{A 1} \rightarrow \chi(2,4,1,3) \rightarrow-4 M \\
& W_{A 2} \rightarrow \chi(4,1,2,3) \rightarrow-M \\
& W_{A 3} \rightarrow \chi(4,3,1,2) \rightarrow-2 M
\end{aligned}
$$

The reflection of $W_{A 2}$, which is not symmetric, will have the structure $\chi(1,4,3,2) \rightarrow$ $-M$.

Beware that, unlike in the asymptotic case, here the exchange $1 \leftrightarrow 4$ is not allowed, since lines 1 and 4 are now neighbours. Furthermore, there is an ambiguity in the choice of the basis. Shifts of all arguments by an integer generate four different chiral functions for $W_{A 1}$ and $W_{A 3}$ and two each for $W_{A 2}$ and for its reflection. A cyclic rotation of the external legs of $W_{A 3}$ generates an additional chiral function. The elements in each of the four classes are equivalent when applied to a length-four state. From each class we have chosen a single representative.

The results after completion of the $D$-algebra for each completely chiral supergraph are given by

$$
\begin{aligned}
& W_{A 1} \rightarrow\left(g^{2} N\right)^{4}\left(I_{4} / 2\right) \chi(2,4,1,3) \rightarrow-2\left(g^{2} N\right)^{4} I_{4} M, \\
& W_{A 2} \rightarrow\left(g^{2} N\right)^{4} I_{2}[\chi(1,4,3,2)+\chi(4,1,2,3)] \rightarrow-2\left(g^{2} N\right)^{4} I_{2} M \\
& W_{A 3} \rightarrow\left(g^{2} N\right)^{4} I_{3} \chi(4,3,1,2) \rightarrow-2\left(g^{2} N\right)^{4} I_{3} M,
\end{aligned}
$$

where $I_{i}$ denote the momentum integrals listed in Table C.8. Then the total contribution from this class reads

$$
\sum W_{A * *} \rightarrow-2\left(g^{2} N\right)^{4}\left(I_{4}+I_{2}+I_{3}\right) M
$$

Graphs with chiral structure $\chi(1,2,3)$ are shown in Figure C.1, while the results of the $D$-algebra for each diagram are summarized schematically in Table C.1; here cancellations between pairs of diagrams, as well as graphs which do not produce any divergence are explicitly indicated. Diagrams which do not cancel and therefore must be fully computed are marked with a $*$. For these relevant graphs we also give explicitly the value of the relative symmetry factor. Graphs with chiral structure $\chi(3,2,1)$, which is the reflection of $\chi(1,2,3)$, contribute exactly in the same manner. We give a summary of the full results for all the structures in Table C.7, where the explicit expressions (3.11) and (4.1) for the chiral structures in the length-four subsector have been used.

So the total contribution from these chiral structures is given by

$$
\sum W_{B * *} \rightarrow-4\left(g^{2} N\right)^{4}\left(I_{7}-I_{9}\right) M
$$


We proceed in the same way for the other structures. Diagrams associated to $\chi(1,3,2)$ are listed in Figure C.2, while Table C.2 contains the results from $D$-algebra.

$$
\sum W_{C * *} \rightarrow-4\left(g^{2} N\right)^{4} I_{6} M
$$

There is one more structure which needs only a vector to be completed to four loops, $\chi(2,1,3)$. The corresponding diagrams and the $D$-algebra results can be read from Figure C.3 and Table C.3. The divergent contribution is 1

$$
\sum W_{D * *} \rightarrow-4\left(g^{2} N\right)^{4} I_{8} M
$$

Next we turn to chiral structures of shorter range. Two of them require two vectors to be completed to four loops: the first one is $\chi(2,1)$, for which diagrams and $D$-algebra results are given in Figure C.4 and Table C.4. Adding the analogous result from graphs with structure $\chi(1,2)$ we obtain

$$
\sum W_{E * *} \rightarrow-4\left(g^{2} N\right)^{4}\left(I_{7}-I_{9}\right) M
$$

The second structure to be completed by two vectors is $\chi(1,3)$ and it is described in Figure C.5 and Table C.5, from which we get

$$
\sum W_{F * *} \rightarrow-4\left(g^{2} N\right)^{4}\left(I_{10}+I_{11}-2 I_{12}\right) M
$$

The last chiral structure to be considered is $\chi(1)$, which is the only one requiring three vectors. The diagrams for this structure are presented in Figure C.6. Using the results summarized in Table C.6 we obtain

$$
\sum W_{G * *} \rightarrow 2\left(g^{2} N\right)^{4}\left(I_{1}-I_{2}\right) M
$$

So up to this point we have obtained the total contribution from each relevant chiral structure. Now we have to substitute the values of the momentum integrals $I_{i}$. In order to obtain the full wrapping contribution to the dilatation operator on the length-four subsector, we have to take the coefficient of the $\frac{1}{\varepsilon}$ pole of these expressions and multiply them by -8 according to eq. (1.2). In the next subsection we describe the computation of these momentum integrals by means of the Gegenbauer polynomial $x$-space technique.

\footnotetext{
${ }^{1}$ In the first version of the paper there is a symmetry factor of 2 missing in this expression. We have found this factor after a revision of our calculation which was suggested by a mismatch between the rational part of our previously found Konishi anomalous dimension and the one computed in [36].
} 


\subsection{Gegenbauer polynomial $x$-space technique}

The integrals with non-trivial numerators which cannot be rephrased in terms of standard scalar integrals, are calculated with the Gegenbauer polynomial $x$-space technique (GPXT) [26], and are independently checked with the method of uniqueness [27] and with the help of MINCER [37], a computer program to compute 3-loop integrals. We have furthermore used the GPXT and MINCER to reproduce the known results for the scalar integrals.

The GPXT is based on the fact that in $x$-space integrals all propagators depend on the difference of only two coordinates and hence can be expanded in a series involving the Gegenbauer polynomials. The non-trivial numerators given by scalar products of several momenta become traceless symmetric products in $x$-space. These products are easily reexpressed in terms of Gegenbauer polynomials. Then the Wick rotated integral is solved by introducing spherical coordinates. As a first step one performs all angular integrations. Due to the orthogonality of the Gegenbauer polynomials, in this way the number of independent summations, stemming from the series expansions, is reduced. Finally, the radial integration is performed, and the remaining summations are evaluated in order to obtain the final result.

In particular the GPXT turns out to be very useful in the following situation:

1. Each angular integration involves maximally two Gegenbauer polynomials. For this it might be necessary to expand products of Gegenbauer polynomials with the same argument in a Clebsch-Gordan series.

2. The integrals contain a vertex on which a large number of propagators end, (in our case provided by the composite operator) which is chosen as the 'root vertex'.

3. By partial integrations the momentum dependence in the numerator can be rearranged such that it is given by a linear combination of scalar products of those momenta which run along propagators directly connected to the root vertex.

4. Only the pole structures of the (logarithmically divergent) integrals are of interest.

Point 1 is essential for solving the integral. It guarantees that the Racah coefficients never appear after having performed the angular integrals. Points 2 to 4 are not essential, but significantly simplify the problem. They reduce the number of nested (infinite) sums.

The first three points are guaranteed if any of the four loops contains at least a single propagator which is connected directly to the root vertex and if the non-trivial numerators do not generate additional vertices which cannot be reduced by a ClebschGordan series expansion. Apart from eventually appearing problems with the non-trivial numerators, the requirement holds for all diagrams which become tree level or one loop if the root vertex is removed. In our four-loop calculation at critical order, this is 
guaranteed for all wrapping diagrams and range-five diagrams. At higher loops, it might only be spoiled by the increasing complexity of the appearing numerators. Therefore, we cannot strictly prove the applicability of GPXT. However, the found numerators even at higher loop order suggest that it might hold. In any case, GPXT will in principle allow for an analytic determination of the pole parts of whole classes of integrals at arbitrary loop order. A practical restriction is only given by the increasing number of domains in the radial integrations.

The fact that we are interested only in the pole structure allows for a dramatical simplification. We can neglect the momentum-dependent exponential factor in the $x$ space integrals, i.e. we can set the external momentum of the propagator-type integrals to zero. We then have to introduce a cutoff $R$ as upper bound of the integration domain. This regularizes the appearing IR divergences in the integral. Since the poles of the logarithmically divergent integrals are independent of the momentum, this procedure does not alter the poles of the integral from which the subdivergences have been subtracted. To ensure this, the IR cutoff has also to be used in the computation of the subdivergences.

GPXT has been proposed in [26], see also [27,38] for examples and some important relations. We will not review the method here, but only stress an important fact which we could not find in the literature. For integrals with more than one derivative on a propagator line, special care has to be taken. The corresponding computations are briefly summarized in Appendix D. We will review the method and the refinements in a separate publication [39].

\subsection{Wrapping dilatation operator}

Having worked out all the integrals presented in Table C.8, we can now write down the wrapping contribution to the four-loop dilatation operator in terms of the chiral functions $\chi$ as follows

$$
\begin{aligned}
D_{4}^{\mathrm{w}}=-8( & 2 \zeta(3) \chi(1)-(3 \zeta(3)-5 \zeta(5))[\chi(1,2)+\chi(2,1)]-(1+3 \zeta(3)-5 \zeta(5)) \chi(1,3) \\
& +(3 \zeta(3)-5 \zeta(5))[\chi(1,2,3)+\chi(3,2,1)]+\frac{7}{6} \chi(1,3,2)+\frac{11}{6} \chi(2,1,3) \\
& -\frac{1}{2}(1-\zeta(3)) \chi(2,4,1,3)+\left(\frac{5}{4}-\zeta(3)\right)[\chi(1,4,3,2)+\chi(4,1,2,3)] \\
& \left.-\left(\frac{1}{2}-\zeta(3)\right) \chi(4,1,3,2)\right)
\end{aligned}
$$


Applying it to our operator basis (3.10) we then have

$$
D_{4}^{\mathrm{w}} \rightarrow-8\left(\frac{17}{2}+18 \zeta(3)-30 \zeta(5)\right) M
$$

\section{The Konishi anomalous dimension at four loops}

We collect the results from the previous three sections. They can be encoded in a dilatation operator at four loops which includes wrapping. It can be obtained by adding together the range-four subtracted dilatation operator (3.9) and the wrapping contribution (4.12). We find

$$
\begin{aligned}
D_{4}^{\mathrm{sub}}+D_{4}^{\mathrm{w}}= & (200-16 \zeta(3)) \chi(1)-(150-24 \zeta(3)+40 \zeta(5))[\chi(1,2)+\chi(2,1)] \\
& +\left(88+8 \epsilon_{3 a}+24 \zeta(3)-40 \zeta(5)\right) \chi(1,3) \\
& +(60-24 \zeta(3)+40 \zeta(5))[\chi(1,2,3)+\chi(3,2,1)] \\
& -\left(\frac{4}{3}-2 \beta-4 \epsilon_{3 a}+4 i \epsilon_{3 b}-2 i \epsilon_{3 c}+4 i \epsilon_{3 d}\right) \chi(1,3,2) \\
& -\left(\frac{20}{3}-2 \beta-4 \epsilon_{3 a}-4 i \epsilon_{3 b}+2 i \epsilon_{3 c}-4 i \epsilon_{3 d}\right) \chi(2,1,3) \\
& +4(1-\zeta(3)) \chi(2,4,1,3)-(10-8 \zeta(3))[\chi(1,4,3,2)+\chi(4,1,2,3)] \\
& -\left(12+2 \beta+4 \epsilon_{3 a}\right) \chi(2,1,3,2)+(4-8 \zeta(3)) \chi(4,1,3,2) .
\end{aligned}
$$

On the basis (3.10) it gives

$$
D_{4}^{\mathrm{sub}}+D_{4}^{\mathrm{w}} \rightarrow(416-96 \zeta(3)+240 \zeta(5)) M .
$$

The non-vanishing eigenvalue is hence found to be

$$
\gamma_{4}=-2496+576 \zeta(3)-1440 \zeta(5)
$$

Restoring the dependence on the coupling constant (1.1), and including also the contributions at lower order [28], the final result for the range-four Konishi-descendant up to four loops reads 2

$$
\gamma=4+12 \lambda-48 \lambda^{2}+336 \lambda^{3}+\lambda^{4}(-2496+576 \zeta(3)-1440 \zeta(5)) .
$$

\footnotetext{
${ }^{2}$ Note that due to a sign change of the $\chi(1)$ contribution in (4.12), the coefficient of the $\zeta(3)$ term differs from the one in the first version of [1].

Moreover, as remarked in the footnote to (4.8), the rational part has been corrected w.r.t to the one in the first version of the present paper. It agrees with the one found in [36].
} 
In the literature there also exist several conjectures on the value of this anomalous dimension $[12,13,28]$ and moreover, as mentioned in the introduction, an explicit component field theory computation [29] has been presented following our superspace calculation. None of these findings shows an agreement. Therefore it is crucial to have an explicit result that can be trusted.

The aim of this paper has been to present all the steps of our calculation. The superspace approach that we have used allowed drastic simplifications: several cancellations were automatic and the number of graphs that one really had to compute was reasonably small. With all the ingredients given in this paper an interested reader might want to reproduce the whole calculation.

\section{Acknowledgements}

This work has been supported in part by INFN and the European Commission RTN program MRTN-CT-2004-005104. 


\section{A Cancellation of non-maximal range-five supergraphs}

In this appendix we use the result of subsection 2.1 to show that the divergent parts of range-five diagrams where one line interacts with the rest of the graph only through a vector line sum up to zero.

Let us consider a generic three-loop, range-four diagram. We want to extend it to range five and four loops by connecting it to a free line from the operator, using a vector propagator. There are three possible structures for the part of the three-loop diagram which directly interacts with the new line. All the possible diagrams coming from each class are shown in figures A.1, A.2 and A.3.

- Class A (Figure A.1) :

As can be seen from the first line of Figure A.4. performing part of the $D$-algebra for diagram $S_{A 1}$ is enough to obtain the same structure of diagram $S_{A 2}$, with a different sign due to the $\square=-p^{2}$ cancelling one propagator. Since the two diagrams have the same color factor, their divergent parts sum up to zero.

Now consider diagrams $S_{A 3}$ and $S_{A 4}$ : they will produce the same result after $D$ algebra, since in a few steps they can be reduced to the same structure (see lines 2 and 3 of Figure A.4). However, the two diagrams have opposite color factors and so their divergent parts cancel.

Diagram $S_{A 5}$ is finite.

- Class B (Figure A.2):

For diagrams $S_{B 1}$ and $S_{B 2}$ the discussion is similar to the one for $S_{A 3}$ and $S_{A 4}$ : a partial $D$-algebra for both diagrams shows that they will produce the same result (see lines 1 and 2 of Figure A.5), and the opposite color factors make the total divergent part vanish.

Diagram $S_{B 3}$ is finite.

- Class C (Figure A.3):

For diagrams $S_{C 1}$ and $S_{C 2}$ we have the same situation as for $S_{A 1}$ and $S_{A 2}$ : diagram $S_{C 1}$ can be reduced to $S_{C 2}$ through the cancellation of a propagator with a $\square$, which gives a minus sign (line 3 of Figure A.5). This difference in sign is responsible for the cancellation of divergent parts, since the two color factors are equal.

Diagram $S_{C 3}$ is finite. 


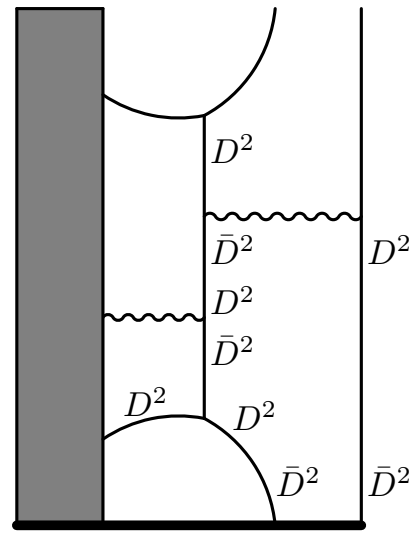

(a) $S_{A 1}$

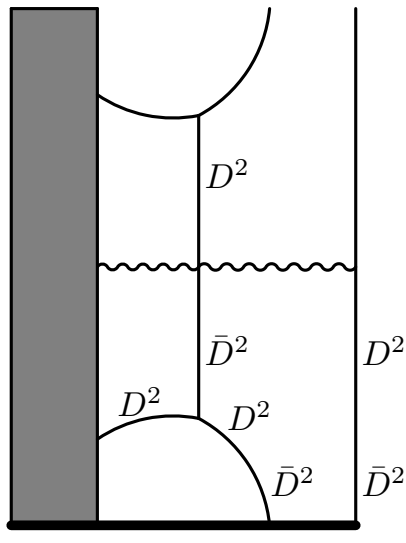

(b) $S_{A 2}$

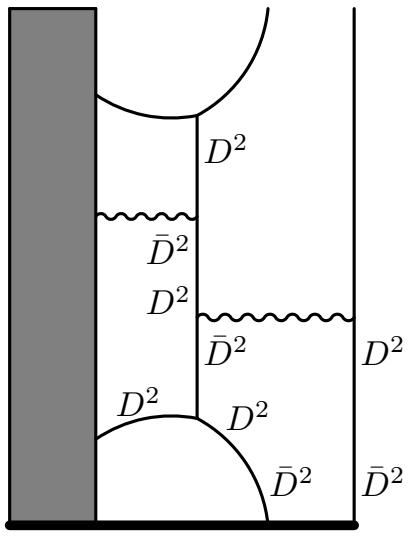

(c) $S_{A 3}$

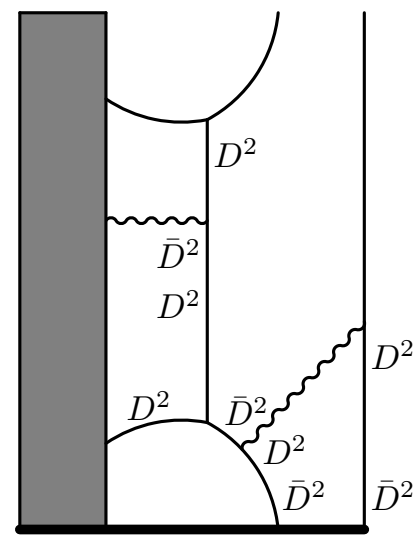

(d) $S_{A 4}$

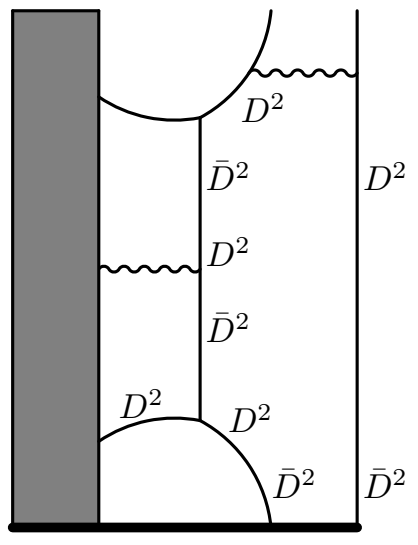

(e) $S_{A 5}$

Figure A.1: Four-loop non-maximal diagrams of class A 


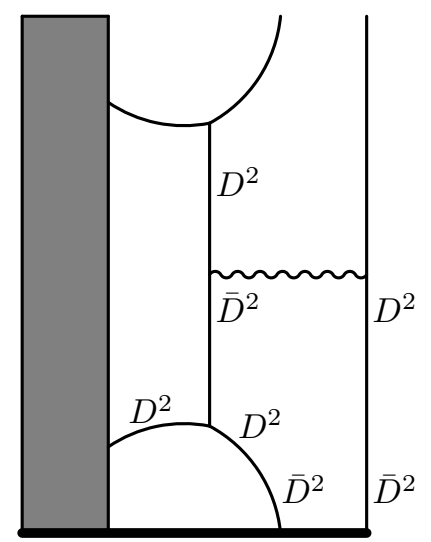

(a) $S_{B 1}$

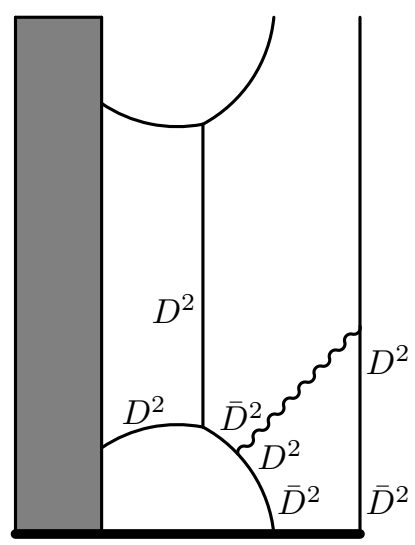

(b) $S_{B 2}$

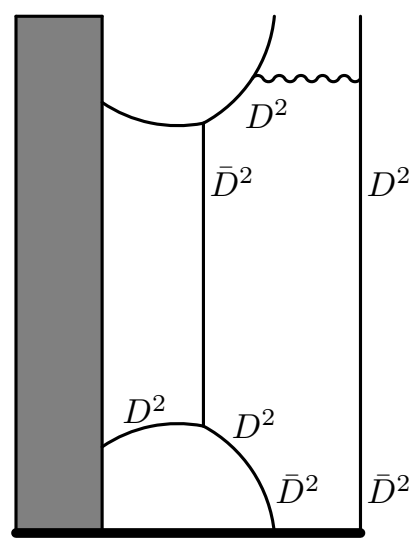

(c) $S_{B 3}$

Figure A.2: Four-loop non-maximal diagrams of class B

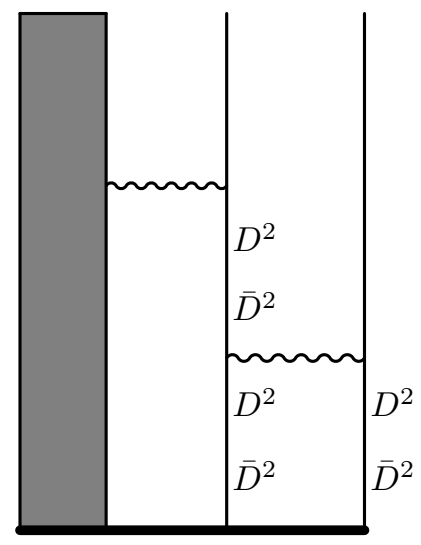

(a) $S_{C 1}$

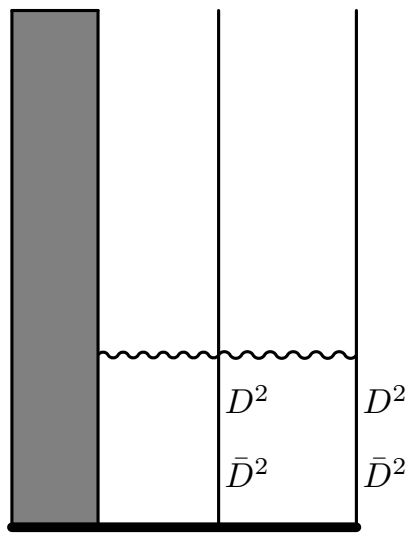

(b) $S_{C 2}$

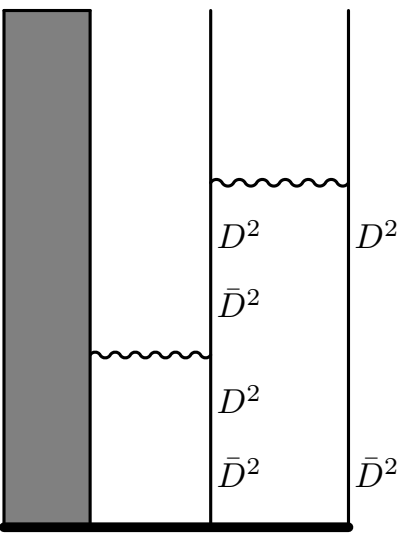

(c) $S_{C 3}$

Figure A.3: Four-loop non-maximal diagrams of class C 

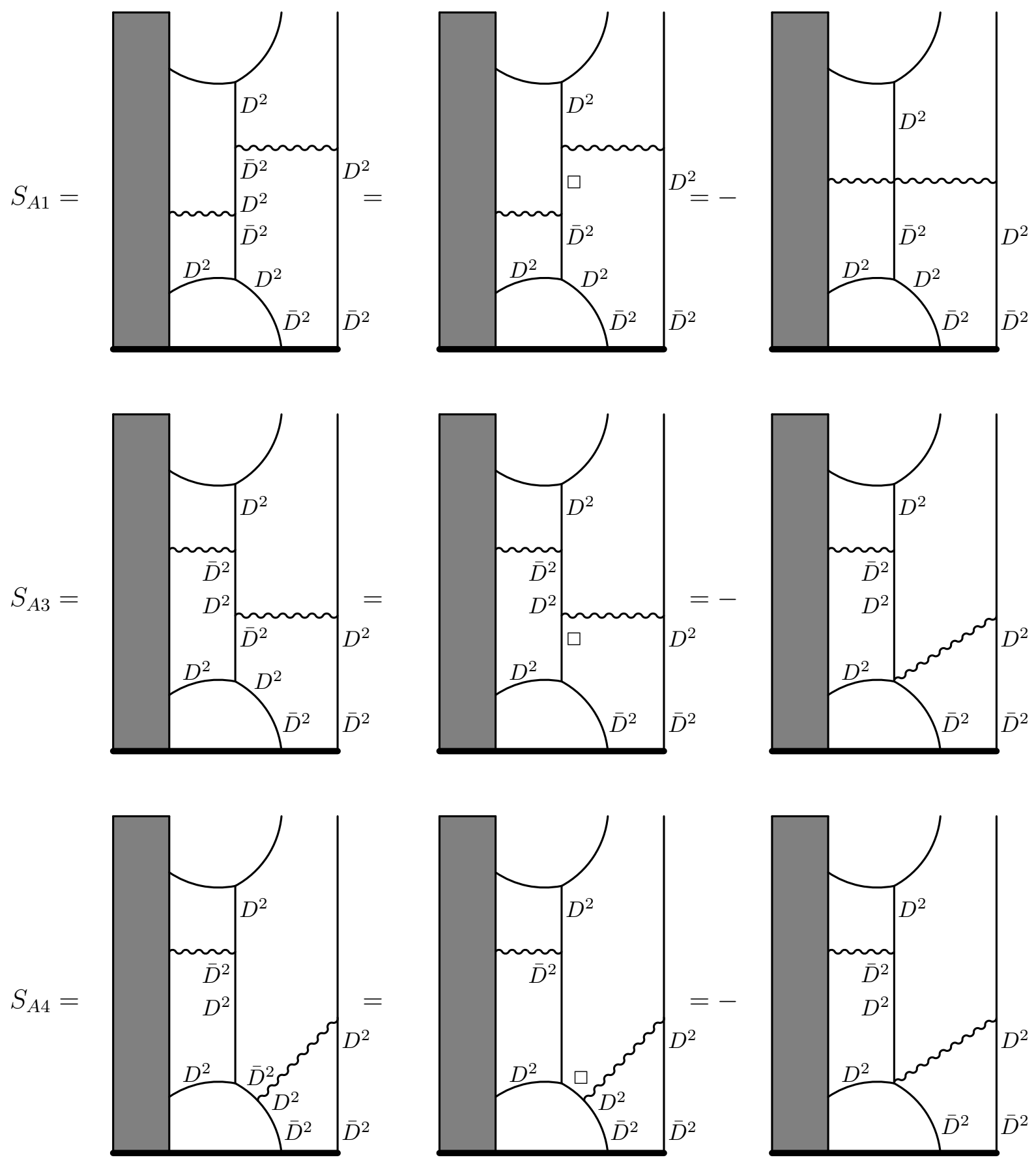

Figure A.4: $D$-algebra steps showing explicit cancellations for class A 

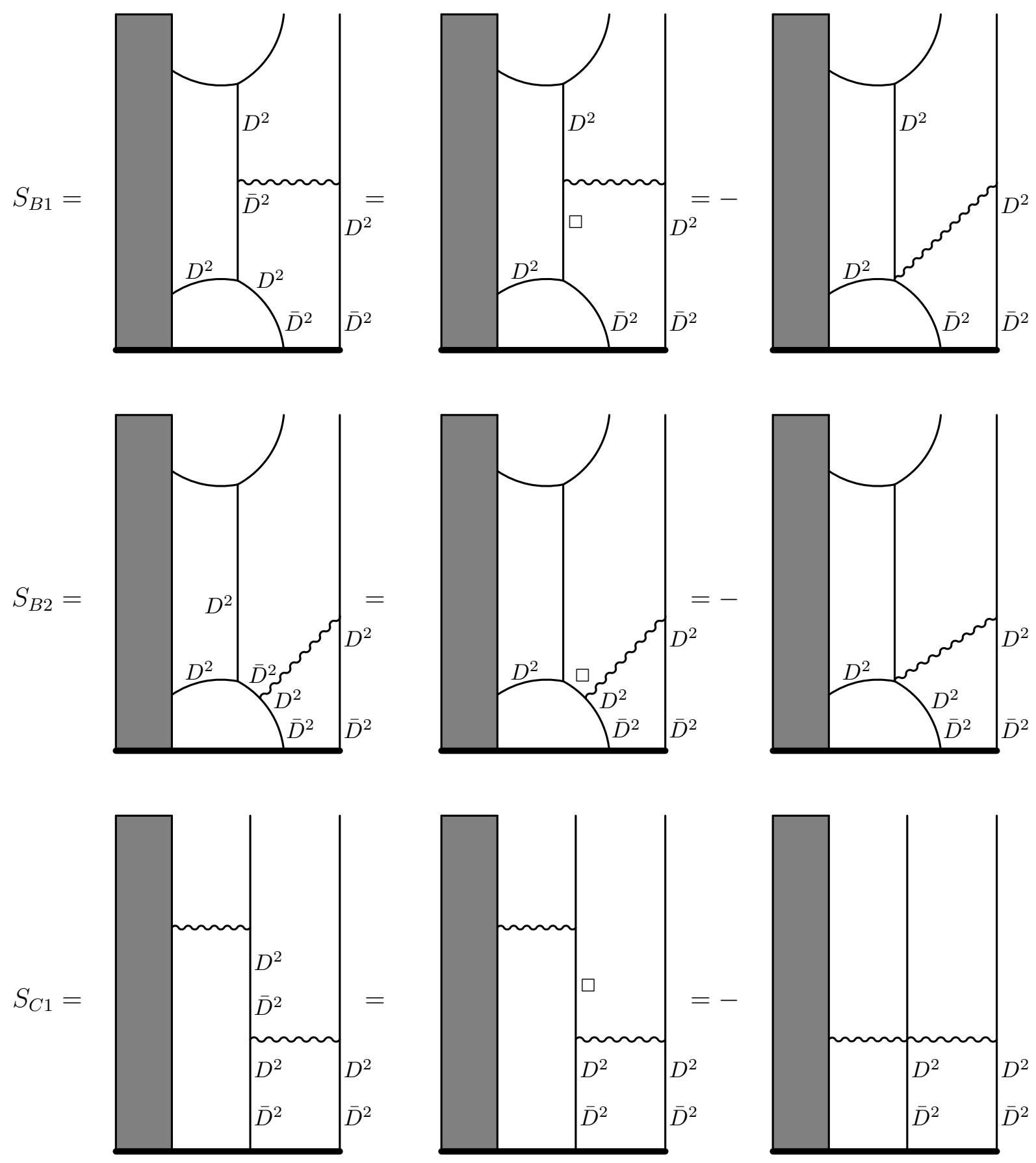

Figure A.5: D-algebra steps showing explicit cancellations for classes B and C 


\section{B Range-five diagrams}

In this appendix we present the results of the computation of range-five diagrams. While this calculation would not be strictly required for the subtraction of range-five interactions from the asymptotic dilatation operator, it has been performed as a check of our procedure. Moreover, it allowed us to compute the values of three out of the four $\epsilon$ coefficients in our scheme.

The completely chiral four-loop diagrams contributing to the asymptotic dilatation operator are shown in Figure B.0. They are all range five, except for the last one which is range four. Each diagram has a different chiral structure, so the corresponding coefficient in the dilatation operator is obtained directly from the value of the diagram.

The diagrams with structures $\chi(2,1,4)$ (which is the reflection of $\chi(1,3,4)$ ), $\chi(1,4,3)$ (reflection of $\chi(1,2,4)$ ) and $\chi(1,4)$ are shown in figures B.1, B.2 and B.3 respectively. The results of $D$-algebra for every diagram in these classes are summarized in tables B.1, B.2 and B.3: cancellations between pairs of supergraphs, as well as diagrams which do not produce any divergent part, are explicitly indicated. In all the other cases, a * indicates that the diagram gives a non-vanishing contribution which must be computed. For these relevant diagrams, we also give the relative symmetry factor. In Table B.4 all these relevant contributions are collected. The integrals $J_{i}$ are given in Table B.5.

Let us define $\mathcal{C}(\chi(\ldots))$ as the coefficient of structure $\chi(\ldots)$ in the dilatation operator (3.6). According to equation (1.2), in order to compute $\mathcal{C}(\chi(\ldots))$ from the sum of the corresponding diagrams, we have to take the coefficient of the $\frac{1}{\varepsilon}$ pole and multiply it by -8 . We find:

- $\mathcal{C}(\chi(1,2,3,4))=-10$, which is a check

- $\mathcal{C}(\chi(3,2,1,4))+\mathcal{C}(\chi(1,4,3,2))=8 / 3-8 / 3=0$

This must be equal to $-2\left(8+2 \epsilon_{3 a}\right)$ from (3.6) . So we obtain $\epsilon_{3 a}=-4$

- $\mathcal{C}(\chi(3,2,1,4))-\mathcal{C}(\chi(1,4,3,2))=8 / 3-(-8 / 3)=16 / 3$

Since this has to be equal to $4 i \epsilon_{3 b}$, we get $\epsilon_{3 b}=-i 4 / 3$

- $\mathcal{C}(\chi(2,4,1,3))=2=18+4 \epsilon_{3 a}$ which is another check.

- $\mathcal{C}(\chi(2,1,3,2))=4-8 \zeta(3)$

This must be equal to $\left(-12-2 \beta-4 \epsilon_{3 a}\right)$, so $\beta=4 \zeta(3)$

- $\mathcal{C}(\chi(2,1,4))+\mathcal{C}(\chi(1,2,4))=-4 / 3-20 / 3=-8$ is a check.

- $\mathcal{C}(\chi(2,1,4))-\mathcal{C}(\chi(1,2,4))=-4 / 3-(-20 / 3)=16 / 3$

This allows to compute $\epsilon_{3 c}$, since from $8 i \epsilon_{3 b}+4 i \epsilon_{3 c}=16 / 3$ we obtain $\epsilon_{3 c}=i 4 / 3$

- $\mathcal{C}(\chi(1,4))=-4$ is a check. 


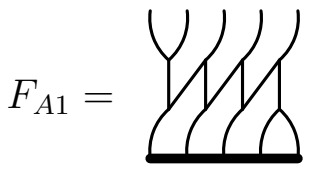

$\chi(1,2,3,4)$

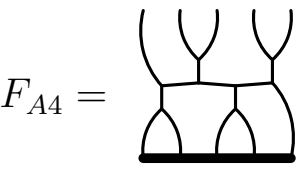

$\chi(2,4,1,3)$

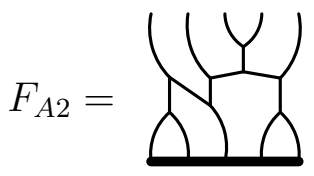

$\chi(3,2,1,4)$

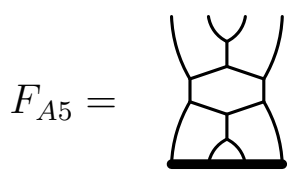

$\chi(2,1,3,2)$

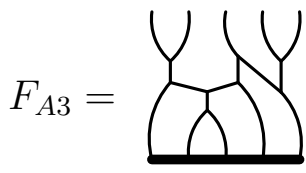

$\chi(1,4,3,2)$

Figure B.0: Diagrams with only chiral interactions
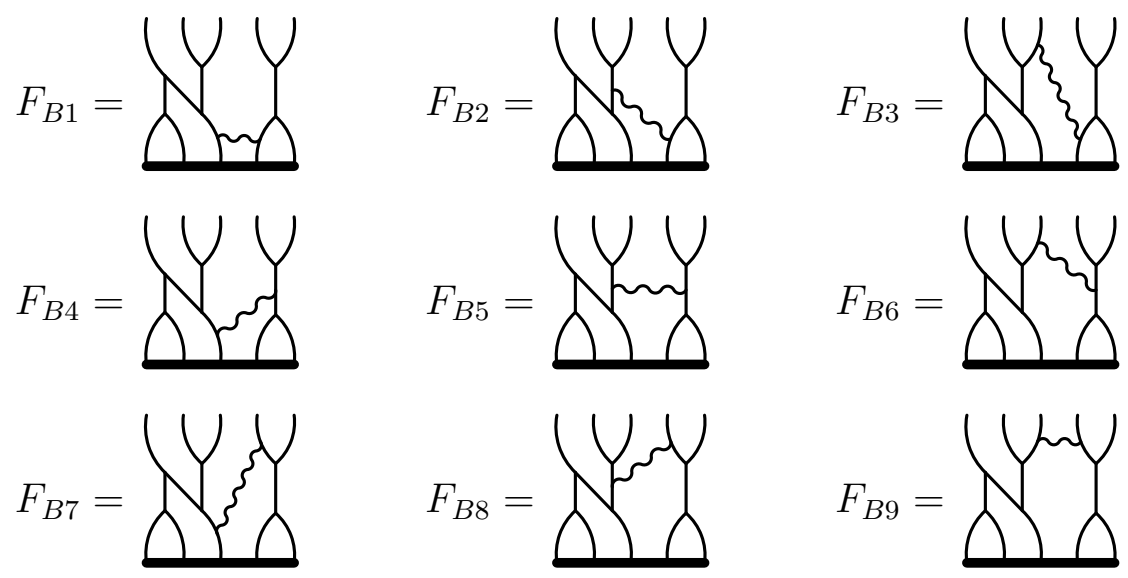

Figure B.1: Diagrams with structure $\chi(2,1,4)$

\begin{tabular}{|c|c|c|c|c|c|c|c|c|c|c|}
\hline$F_{B 1}$ & $\rightarrow$ & $*$ & 1 & $F_{B}$ & $\rightarrow$ & $*$ & 1 & $F_{B 7}$ & $\rightarrow$ & $-F_{B 8}$ \\
\hline$F_{B 2}$ & $\rightarrow$ & $*$ & 1 & $F_{B 5}$ & $\rightarrow$ & finite & & $F_{B 8}$ & $\rightarrow$ & $-F_{B 7}$ \\
\hline$F_{B 3}$ & $\rightarrow$ & $-F_{B 6}$ & & $F_{B 6}$ & $\rightarrow$ & $-F_{B 3}$ & & $F_{B 9}$ & $\rightarrow$ & finite \\
\hline
\end{tabular}

Table B.1: Results of $D$-algebra for diagrams with structure $\chi(2,1,4)$ 

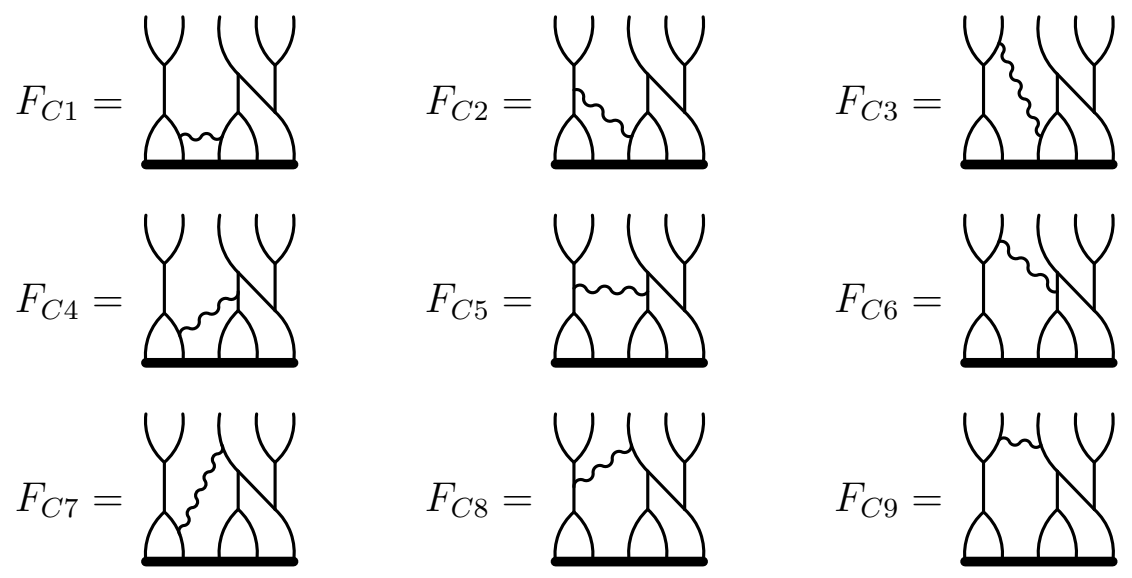

Figure B.2: Diagrams with structure $\chi(1,4,3)$

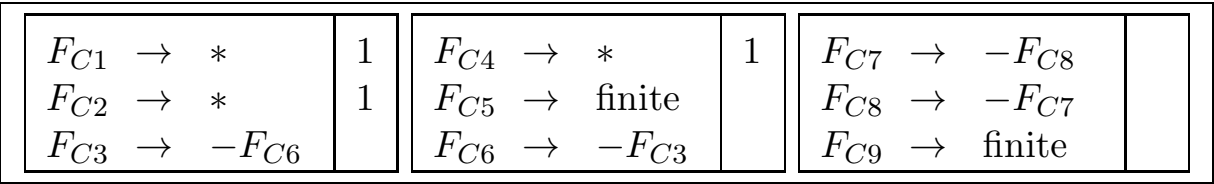

Table B.2: Results of $D$-algebra for diagrams with structure $\chi(1,4,3)$ 

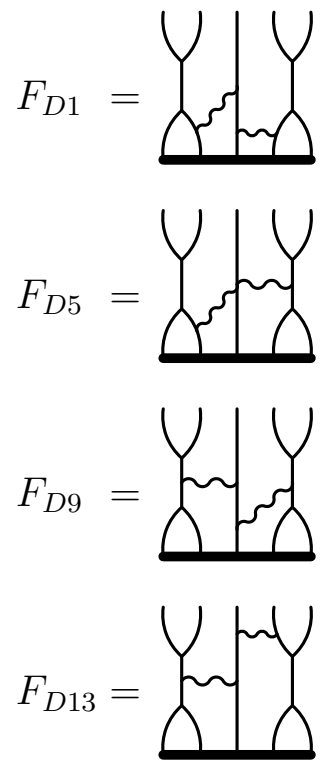
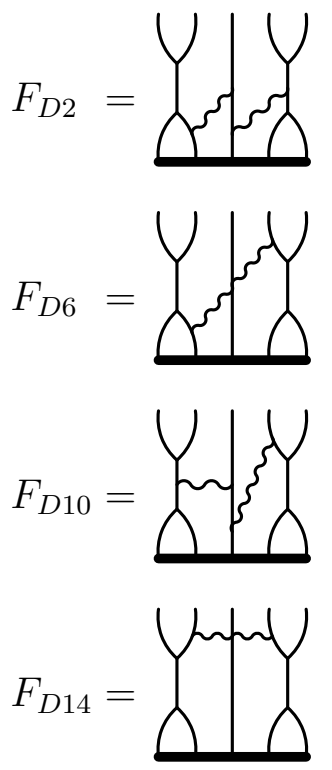
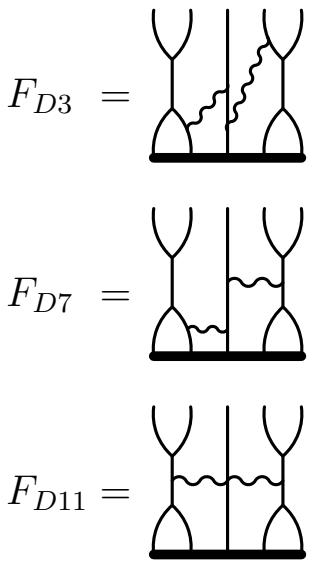
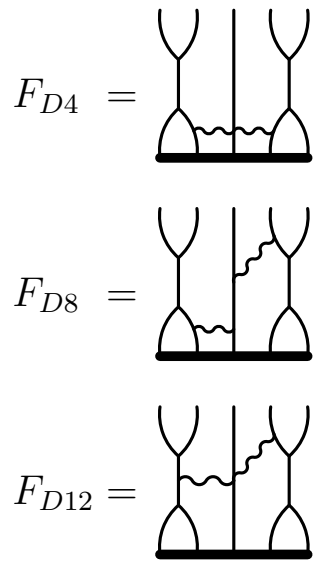

Figure B.3: Diagrams with structure $\chi(1,4)$

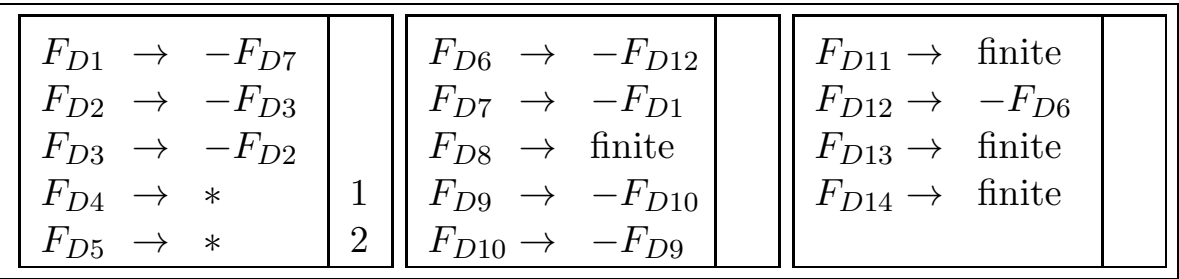

Table B.3: Results of $D$-algebra for diagrams with structure $\chi(1,4)$ 


\begin{tabular}{|c|c|}
\hline$F_{A 1} \rightarrow\left(g^{2} N\right)^{4} J_{1} \chi(1,2,3,4)$ \\
$F_{A 2} \rightarrow\left(g^{2} N\right)^{4} J_{2} \chi(3,2,1,4)$ \\
$F_{A 3} \rightarrow\left(g^{2} N\right)^{4} J_{3} \chi(1,4,3,2)$ \\
$F_{A 4} \rightarrow\left(g^{2} N\right)^{4} J_{4} \chi(2,4,1,3)$ \\
$F_{A 5} \rightarrow\left(g^{2} N\right)^{4} J_{5} \chi(2,1,3,2)$ \\
\hline \hline$F_{B 1} \rightarrow\left(g^{2} N\right)^{4}\left(J_{1}+J_{2}-2 J_{6}\right) \chi(2,1,4)$ \\
$F_{B 2} \rightarrow-\left(g^{2} N\right)^{4} J_{1} \chi(2,1,4)$ \\
$F_{B 4} \rightarrow-\left(g^{2} N\right)^{4} J_{2} \chi(2,1,4)$ \\
\hline \multicolumn{2}{|c|}{$F_{B * *} \rightarrow-2\left(g^{2} N\right)^{4} J_{6} \chi(2,1,4)$} \\
\hline \hline$F_{C 1} \rightarrow\left(g^{2} N\right)^{4}\left(J_{1}+J_{3}-2 J_{7}\right) \chi(1,2,4)$ \\
$F_{C 2} \rightarrow-\left(g^{2} N\right)^{4} J_{1} \chi(1,2,4)$ \\
$F_{C 4} \rightarrow-\left(g^{2} N\right)^{4} J_{3} \chi(1,2,4)$ \\
\hline$\sum F_{C * *} \rightarrow-2\left(g^{2} N\right)^{4} J_{7} \chi(1,2,4)$ \\
\hline \hline$F_{D 4} \rightarrow-2\left(g^{2} N\right)^{4}\left(J_{1}+J_{8}\right) \chi(1,4)$ \\
$F_{D 5} \rightarrow 2\left(g^{2} N\right)^{4} J_{1} \chi(1,4)$ \\
\hline \\
$\sum F_{D * *} \rightarrow-2\left(g^{2} N\right)^{4} J_{8} \chi(1,4)$ \\
\hline
\end{tabular}

Table B.4: Summary of $D$-algebra results. 


$$
\begin{aligned}
& J_{1}=\frac{1}{(4 \pi)^{8}}\left(-\frac{1}{24 \varepsilon^{4}}+\frac{1}{4 \varepsilon^{3}}-\frac{19}{24 \varepsilon^{2}}+\frac{5}{4 \varepsilon}\right) \\
& =\frac{1}{(4 \pi)^{8}}\left(-\frac{1}{8 \varepsilon^{4}}+\frac{1}{3 \varepsilon^{3}}-\frac{5}{24 \varepsilon^{2}}-\frac{1}{3 \varepsilon}\right) \\
& =\frac{1}{(4 \pi)^{8}}\left(-\frac{1}{8 \varepsilon^{4}}+\frac{1}{2 \varepsilon^{3}}-\frac{7}{8 \varepsilon^{2}}+\frac{1}{3 \varepsilon}\right) \\
& =\frac{1}{(4 \pi)^{8}}\left(-\frac{5}{24 \varepsilon^{4}}+\frac{5}{12 \varepsilon^{3}}+\frac{1}{24 \varepsilon^{2}}-\frac{1}{4 \varepsilon}\right) \\
& =\frac{1}{(4 \pi)^{8}}\left(-\frac{1}{4 \pi)^{8}}\left(\frac{1}{4 \varepsilon^{3}}\right)\right. \\
& =
\end{aligned}
$$

Table B.5: Loop integrals for range-five diagrams. The arrows indicate contracted spacetime derivatives. 


\section{Four-loop wrapping diagrams with vectors}
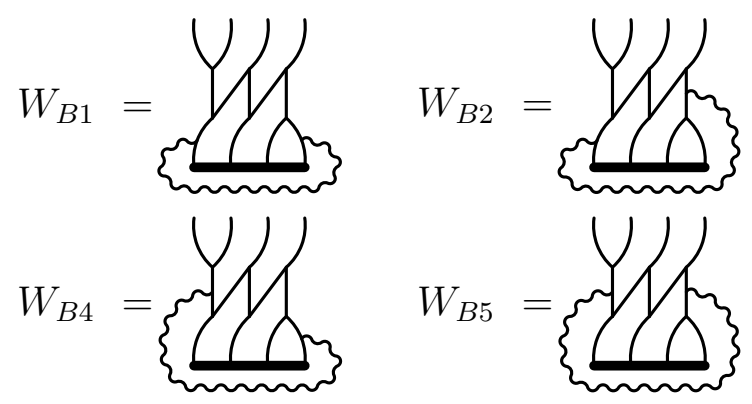

$W_{B 7}=$

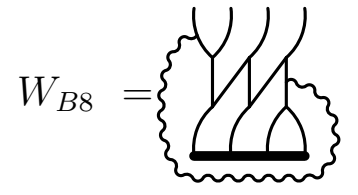

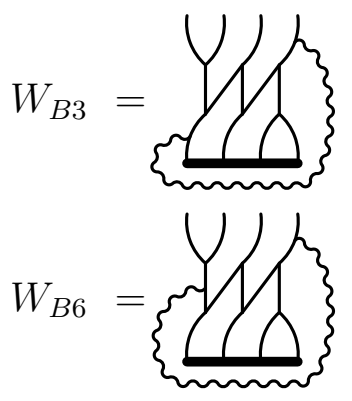

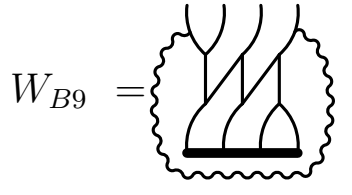

Figure C.1: Wrapping diagrams with chiral structure $\chi(1,2,3)$

\begin{tabular}{|l|l|}
\hline$W_{B 1} \rightarrow *$ & 1 \\
$W_{B 2} \rightarrow *$ & 1 \\
$W_{B 3} \rightarrow *$ & 1
\end{tabular} \mid \begin{tabular}{ll|l|l|l|l|}
$W_{B 4} \rightarrow *$ & 1 \\
$W_{B 5} \rightarrow-W_{B 6}$ & \\
$W_{B 6} \rightarrow-W_{B 5}$ & \\
$W_{B 7} \rightarrow$ finite & \\
$W_{B 8} \rightarrow$ finite & \\
$W_{B 9} \rightarrow$ finite & \\
\hline
\end{tabular}

Table C.1: Results of $D$-algebra for diagrams with structure $\chi(1,2,3)$ 

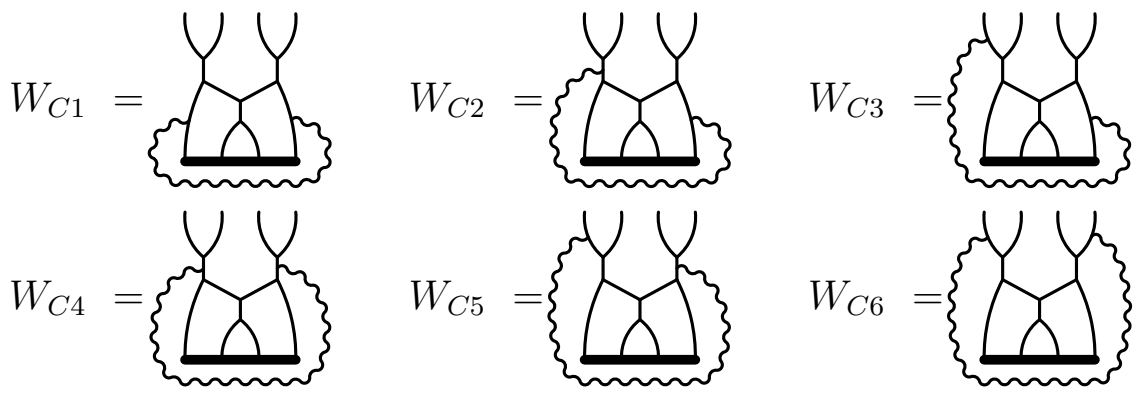

Figure C.2: Wrapping diagrams with chiral structure $\chi(1,3,2)$

\begin{tabular}{|l|l|l|l|l|}
\hline$W_{C 1} \rightarrow *$ & 1 \\
$W_{C 2} \rightarrow *$ & 2 \\
$W_{C 3} \rightarrow-W_{C 5}$ & \\
$W_{C 5} \rightarrow$ finite & \\
$W_{C 5} \rightarrow-W_{C 3}$ & \\
$W_{C 6} \rightarrow$ finite & \\
\hline
\end{tabular}

Table C.2: Results of $D$-algebra for diagrams with structure $\chi(1,3,2)$
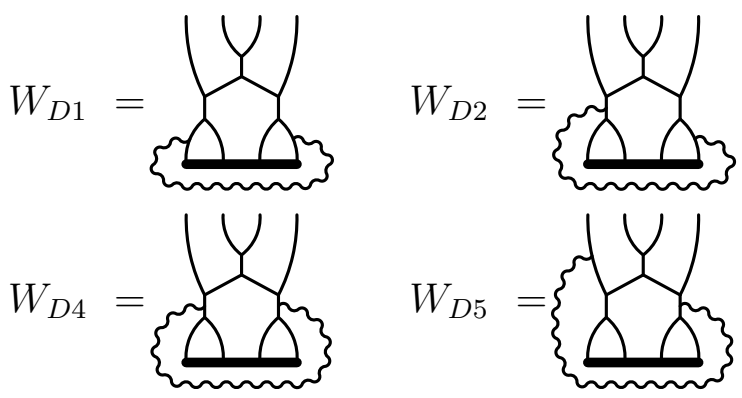

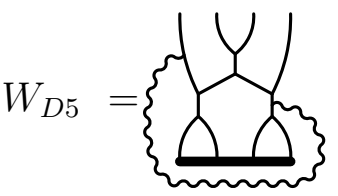

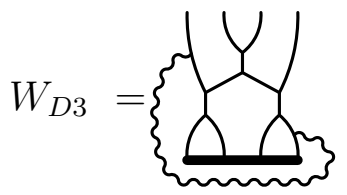

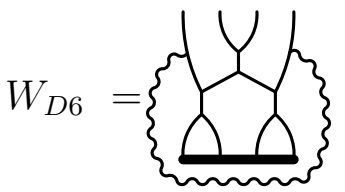

Figure C.3: Wrapping diagrams with chiral structure $\chi(2,1,3)$

\begin{tabular}{|l|l|l|l|}
\hline$W_{D 1} \rightarrow *$ & 1 \\
$W_{D 2} \rightarrow *$ & 2 \\
$W_{D 3} \rightarrow-W_{D 5}$ & \\
$W_{D 4} \rightarrow$ finite & \\
$W_{D 5} \rightarrow-W_{D 3}$ & \\
$W_{D 6}$ & $\rightarrow$ finite \\
\hline
\end{tabular}

Table C.3: Results of $D$-algebra for diagrams with structure $\chi(2,1,3)$ 


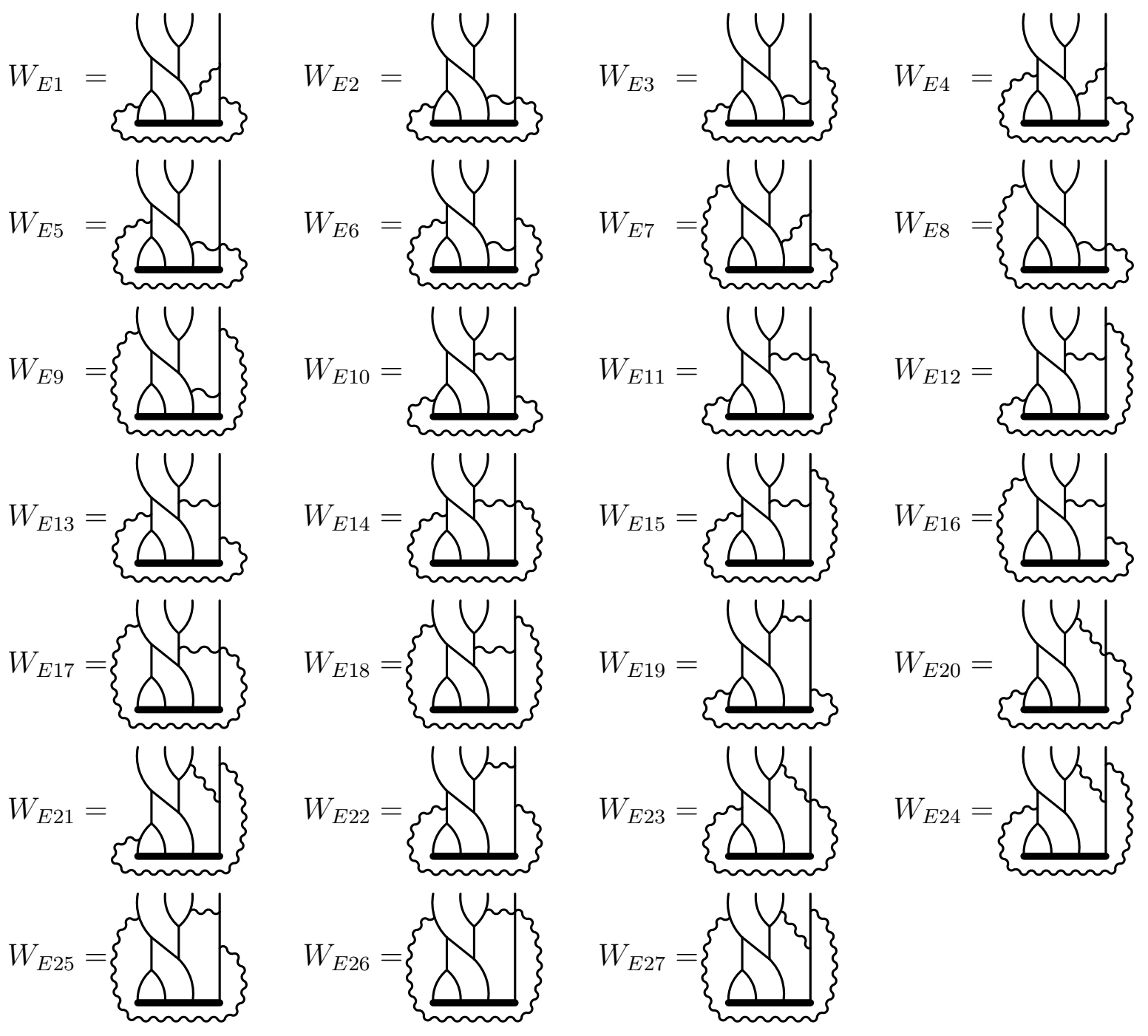

Figure C.4: Wrapping diagrams with chiral structure $\chi(2,1)$

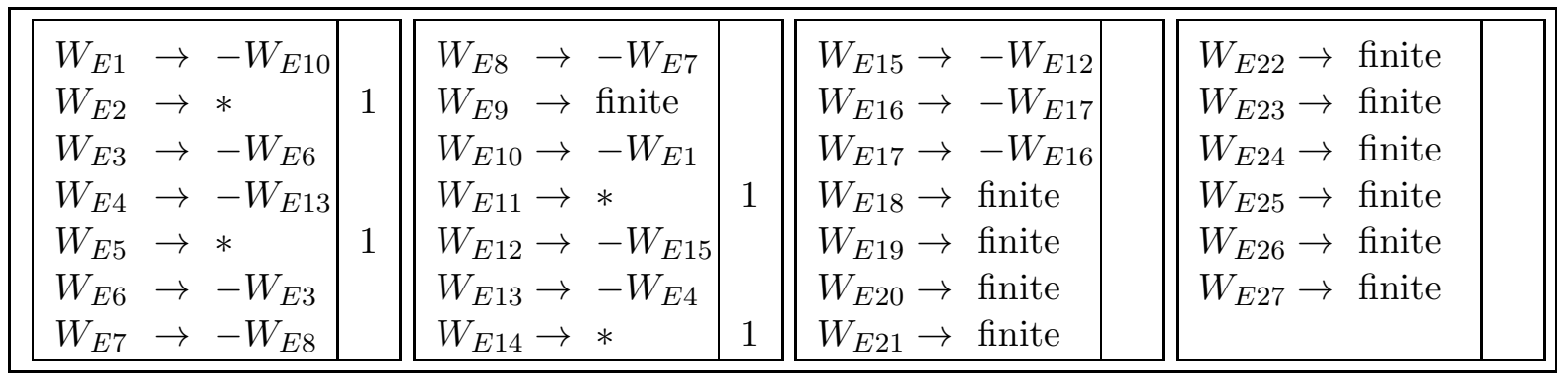

Table C.4: Results of $D$-algebra for diagrams with structure $\chi(2,1)$ 


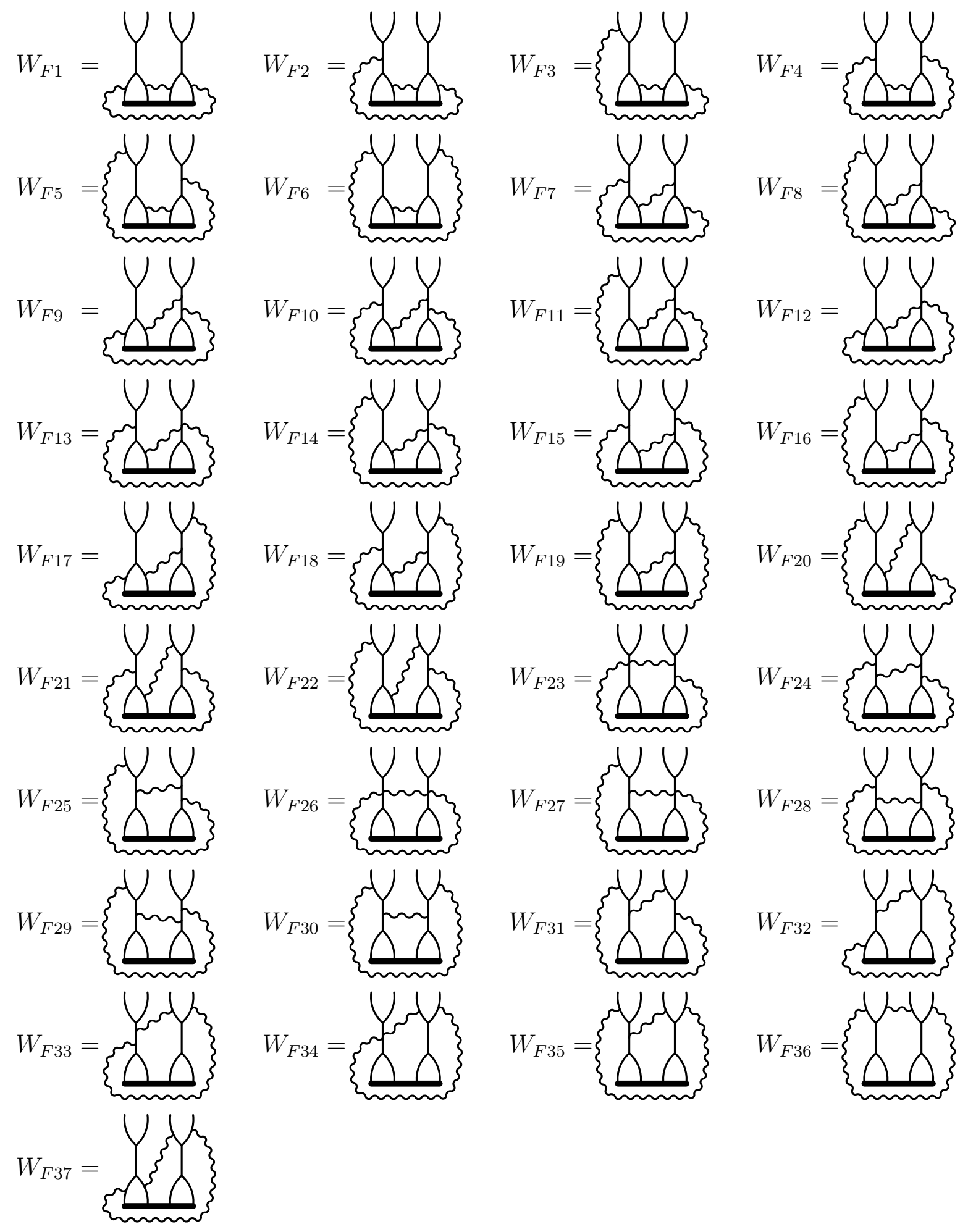

Figure C.5: Wrapping diagrams with chiral structure $\chi(1,3)$ 


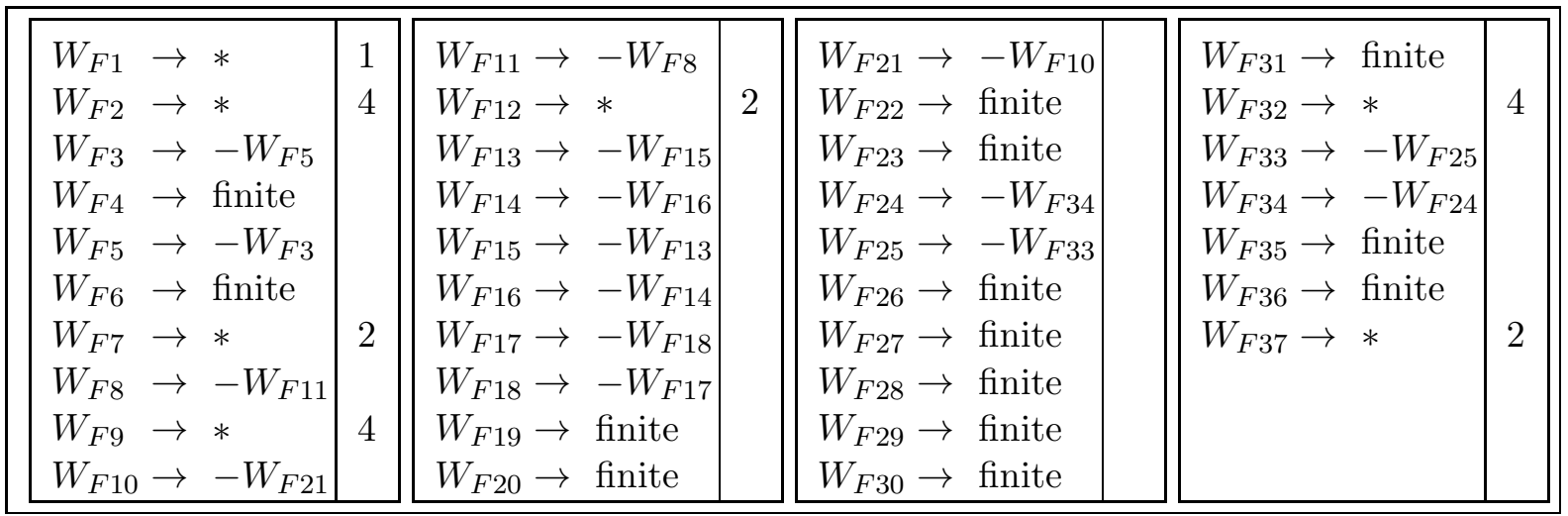

Table C.5: Results of $D$-algebra for diagrams with structure $\chi(1,3)$ 


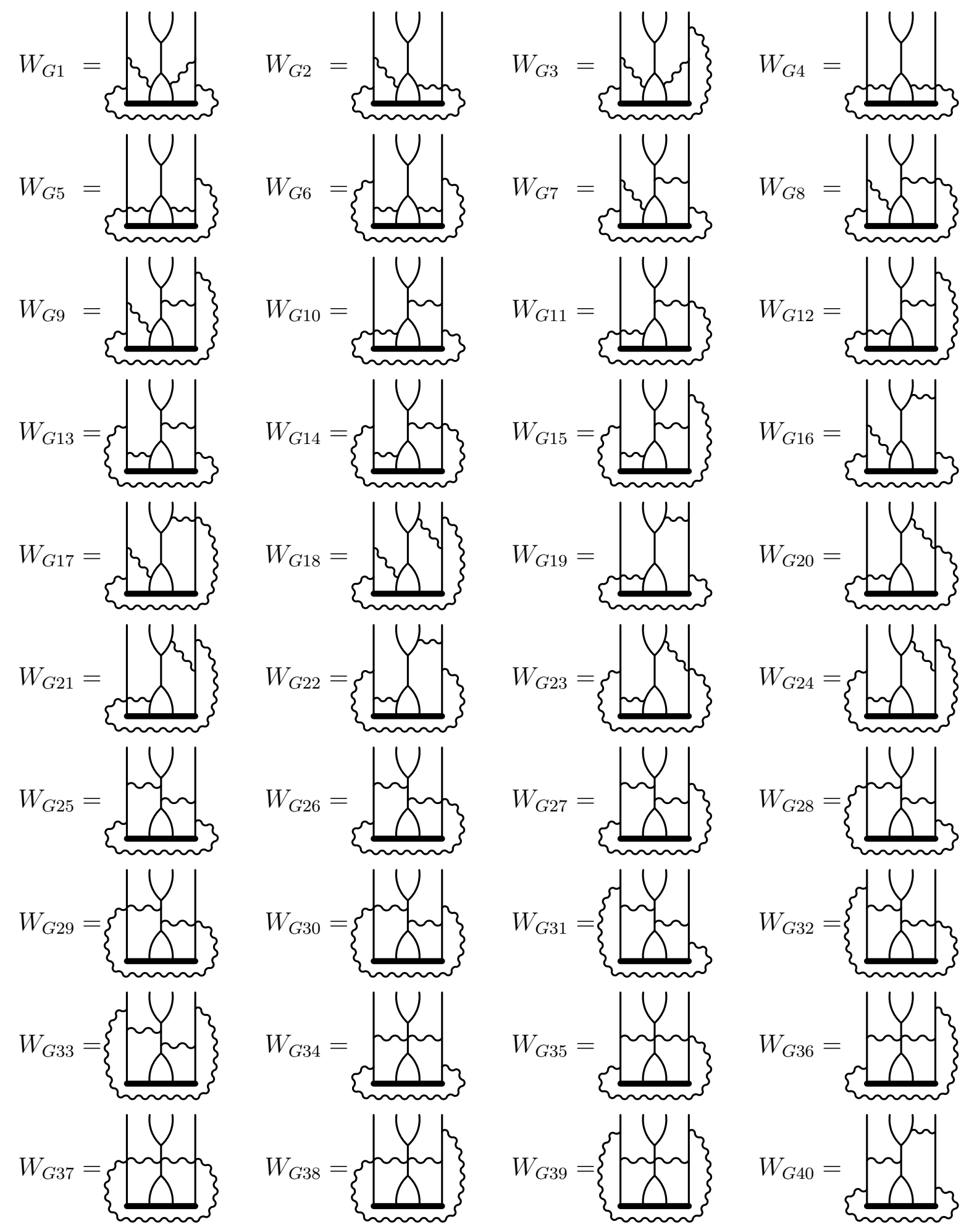

Figure C.6: Wrapping diagrams with chiral structure $\chi(1)$ 

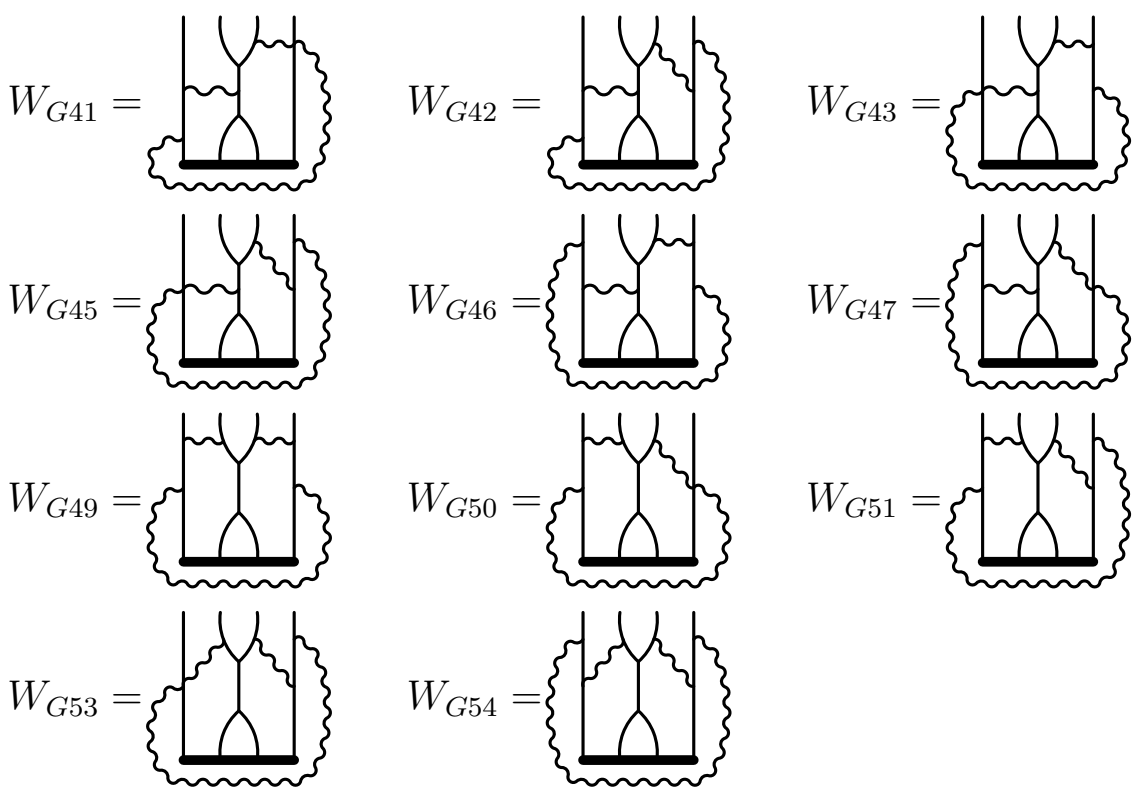
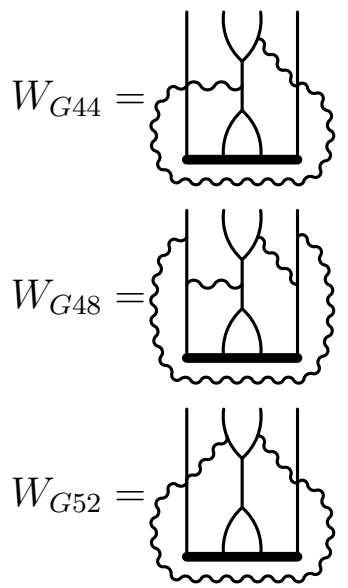

Figure C.6: Wrapping diagrams with chiral structure $\chi(1)$ (continued)

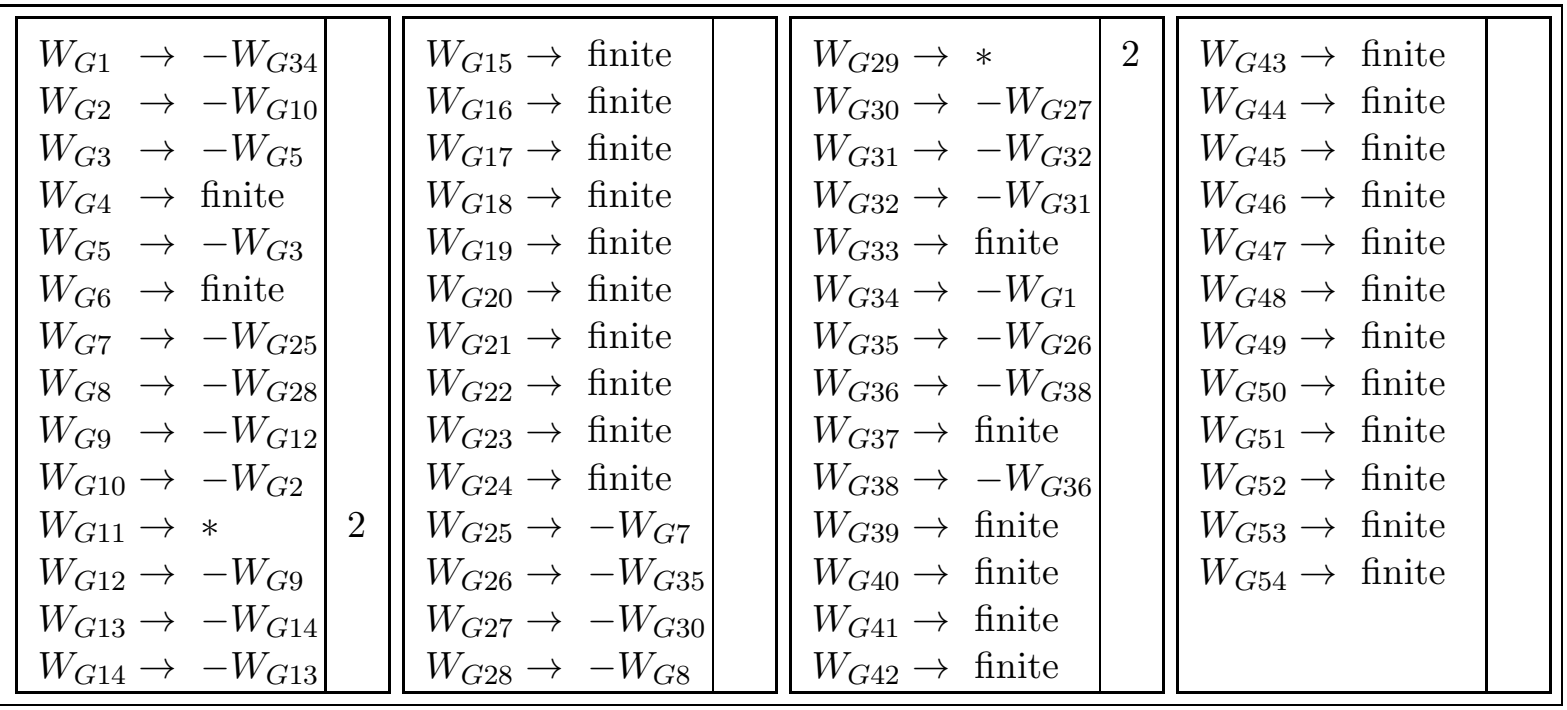

Table C.6: Results of $D$-algebra for diagrams with structure $\chi(1)$ 


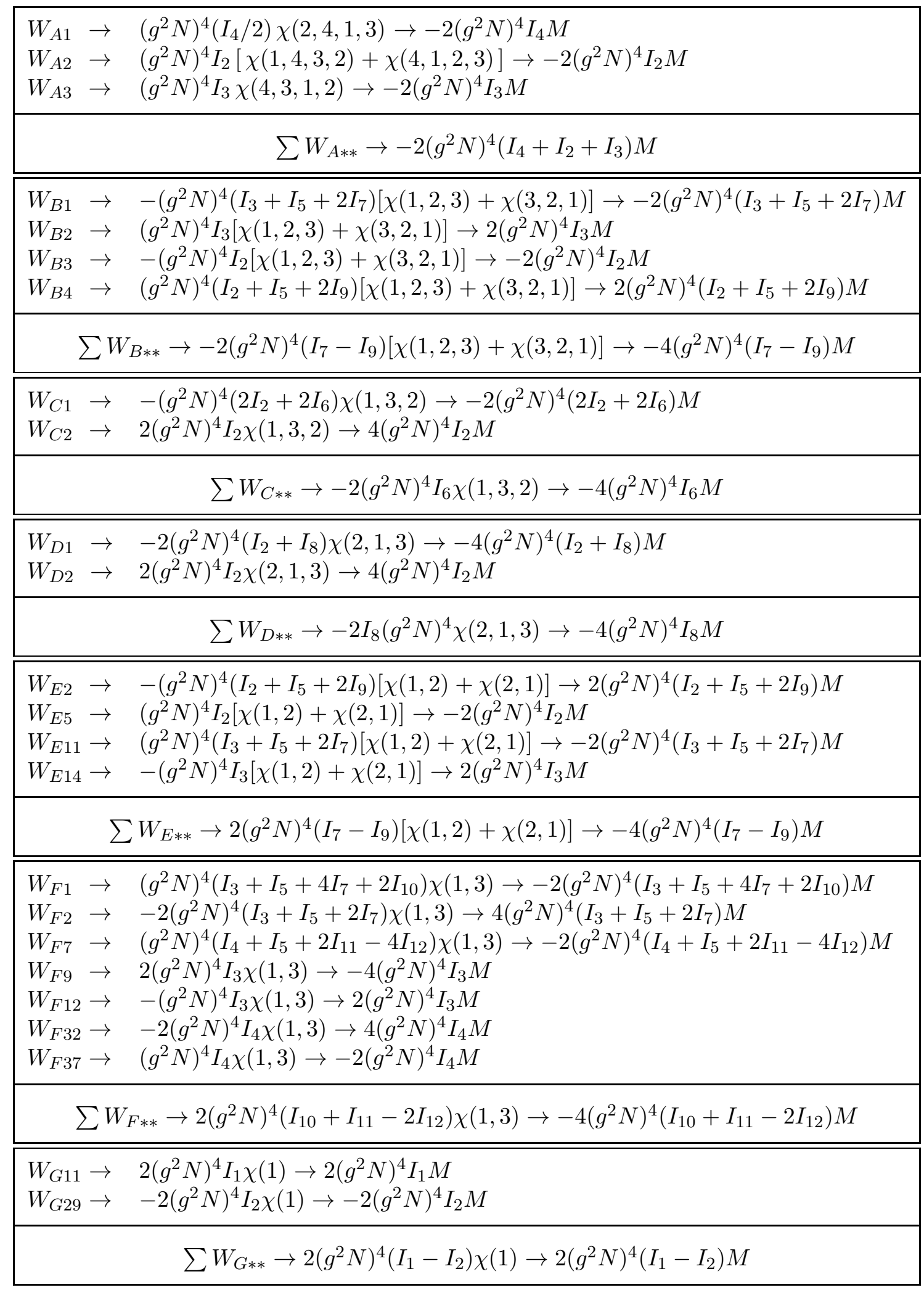

Table C.7: Wrapping contributions at four loops 


$$
\begin{aligned}
& I_{1}=J_{1}=\frac{1}{(4 \pi)^{8}}\left(-\frac{1}{24 \varepsilon^{4}}+\frac{1}{4 \varepsilon^{3}}-\frac{19}{24 \varepsilon^{2}}+\frac{5}{4 \varepsilon}\right) \\
& I_{3}=J_{5}=\frac{1}{(4 \pi)^{8}}\left(-\frac{1}{24 \varepsilon^{4}}+\frac{1}{4 \varepsilon^{3}}-\frac{19}{24 \varepsilon^{2}}+\frac{1}{\varepsilon}\left(\frac{5}{4}-\zeta(3)\right)\right) \\
& =\frac{1}{(4 \pi)^{8}}\left(-\frac{1}{12 \varepsilon^{4}}+\frac{1}{3 \varepsilon^{3}}-\frac{5}{12 \varepsilon^{2}}-\frac{1}{\varepsilon}\left(\frac{1}{2}-\zeta(3)\right)\right) \\
& =\frac{1}{(4 \pi)^{8}} \frac{1}{\varepsilon} 5 \zeta(5)
\end{aligned}
$$

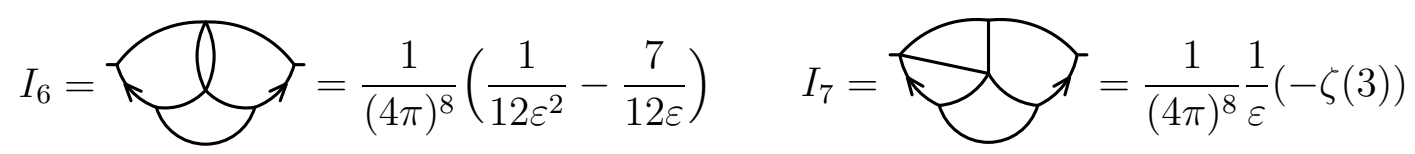

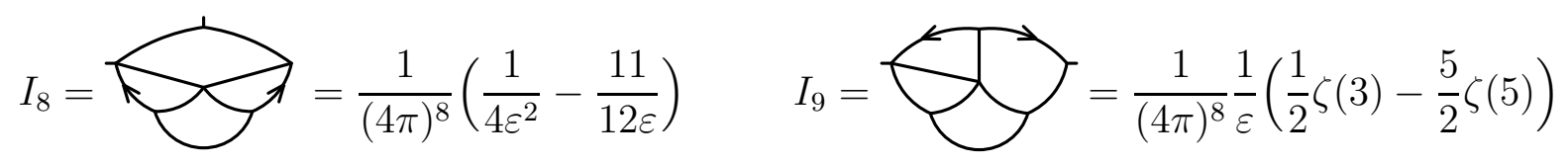$$
I_{10}=\frac{1}{(4 \pi)^{8}} \frac{1}{\varepsilon}\left(-\frac{1}{2}-\frac{1}{2} \zeta(3)+\frac{5}{2} \zeta(5)\right)
$$$$
I_{11}=-\frac{1}{(4 \pi)^{8}} \frac{1}{\varepsilon}\left(-\frac{1}{4}-\frac{3}{2} \zeta(3)+\frac{5}{2} \zeta(5)\right)
$$$$
I_{12}=\overbrace{7}=\frac{1}{(4 \pi)^{8}} \frac{1}{\varepsilon}\left(-\frac{1}{8}-\frac{1}{4} \zeta(3)+\frac{5}{4} \zeta(5)\right)
$$

Table C.8: Loop integrals for 4-loop wrapping diagrams. The arrows of the same type indicate contracted spacetime derivatives 


\section{Integral calculation via GPXT}

We use the Gegenbauer polynomial $x$-space technique (GPXT) to compute the fourloop propagator-type logarithmic divergent integrals. The integrals are dimensionally regularized in $D=4-2 \varepsilon=2(\lambda+1)$ spacetime dimensions, i.e. the parameter $\lambda$ assumes the value

$$
\lambda=1-\varepsilon .
$$

We briefly describe our notation. Parentheses around spacetime indices denote a traceless symmetric product. For two vectors it is defined as

$$
x_{1}^{(\mu} x_{2}^{\nu)}=x_{1}^{\mu} x_{2}^{\nu}-\frac{x_{1} \cdot x_{2}}{D} g^{\mu \nu} .
$$

In $x$-space the integrals can be solved by an expansion of the propagators and traceless

symmetric products in the numerator in terms of Gegenbauer polynomials $C_{n}^{\lambda}$ as follows

$$
\begin{gathered}
\frac{1}{\left(x_{1}-x_{2}\right)^{2 \lambda}}=\frac{1}{\max _{12}^{\lambda}} \sum_{n=0}^{\infty} C_{n}^{\lambda}\left(\hat{x}_{1} \cdot \hat{x}_{2}\right)\left(\frac{\min _{12}}{\max _{12}}\right)^{\frac{n}{2}}, \\
x_{1}^{\left(\mu_{1} \ldots \mu_{n}\right)} x_{2}^{\left(\mu_{1} \ldots \mu_{n}\right)}=\frac{n ! \Gamma(\lambda)}{2^{n} \Gamma(n+\lambda)} C_{n}^{\lambda}\left(\hat{x}_{1} \cdot \hat{x}_{2}\right)\left(r_{1} r_{2}\right)^{\frac{n}{2}} .
\end{gathered}
$$

where $r_{i}=x_{i}^{2}$ denote the radial coordinates in Euclidean space, and $\hat{x}_{i}$ is the corresponding unit vector in the direction of $x_{i}$. We have also abbreviated

$$
\min _{i j}=\min \left(r_{i}, r_{j}\right), \quad \max _{i j}=\max \left(r_{i}, r_{j}\right) .
$$

The Gegenbauer polynomials fulfill

$$
C_{n}^{\lambda}(1)=\frac{\Gamma(n+2 \lambda)}{n ! \Gamma(2 \lambda)} .
$$

Appearing products of two Gegenbauer polynomials with the same argument have to be expanded according to the Clebsch-Gordan series

$$
\begin{aligned}
C_{m}^{\lambda}(x) C_{n}^{\lambda}(x) & =\sum_{\substack{i=|m-n| \\
\frac{i+m+n}{2} \in \mathbb{N}}}^{m+n} D_{\lambda}(m, n, i) C_{i}^{\lambda}(x), \\
D_{\lambda}(m, n, i) & =\frac{i !(i+\lambda) \Gamma\left(\frac{m+n+i}{2}+2 \lambda\right)}{\Gamma(\lambda)^{2} \Gamma\left(\frac{m+n+i}{2}+\lambda+1\right) \Gamma(i+2 \lambda)} \frac{\Gamma\left(\frac{-m+n+i}{2}+\lambda\right) \Gamma\left(\frac{m-n+i}{2}+\lambda\right) \Gamma\left(\frac{m+n-i}{2}+\lambda\right)}{\Gamma\left(\frac{-m+n+i}{2}+1\right) \Gamma\left(\frac{m-n+2}{2}+1\right) \Gamma\left(\frac{m+n-2}{2}+1\right)},
\end{aligned}
$$


where it is important to notice that for positive $\lambda$ the coefficients $D_{\lambda}(m, n, i)$ never become singular.

For compact notation, we introduce the normalization factor

$$
N_{\lambda}(P, L)=\frac{\Gamma(\lambda)^{P}}{\left(2^{2 L} \pi^{P}\right)^{1+\lambda}} \frac{1}{2^{P-L}} \Omega_{D-1}^{P-L},
$$

which depends on the number of propagators $P$ and number of loops $L$. It arises when the momentum integral is transformed via a Fourier transformation into $x$-space, where the integral measure is then rewritten in polar coordinates according to

$$
\mathrm{d}^{D} x=\frac{1}{2} \Omega_{D-1} r^{\lambda} \mathrm{d} r \mathrm{~d} \hat{x}
$$

Here $\hat{x}$ is the unit angular vector, and the volume of the $D$-1-dimensional unit sphere is given by

$$
\Omega_{D-1}=\frac{2 \pi^{\frac{D}{2}}}{\Gamma\left(\frac{D}{2}\right)}
$$

\section{D.1 Sample calculation of $I_{6}$ with two derivatives}

As an example for the computation of the pole part of integrals with two derivatives we show how to obtain $I_{6}$ of Table C.8. This integral contains five propagators which do not end at the root vertex.

Shifting the derivatives to the root vertex, the required integral can be rewritten as

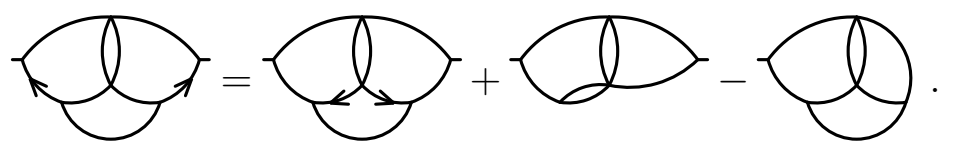

We hence have to compute the pole part of the integral

$$
\Longrightarrow=-\lambda^{2} \frac{\Gamma(\lambda)^{9}}{\left(2^{8} \pi^{9}\right)^{1+\lambda}} \int \frac{\mathrm{d}^{D} x_{1} \mathrm{~d}^{D} x_{2} \mathrm{~d}^{D} x_{3} \mathrm{~d}^{D} x_{4} \mathrm{~d}^{D} x_{5} x_{4} \cdot x_{5} \mathrm{e}^{2 i p \cdot\left(x_{3}-x_{1}\right)}}{x_{2}^{4 \lambda}\left(x_{4}^{2} x_{5}^{2}\right)^{1+\lambda}\left(\Delta_{12}^{2} \Delta_{23}^{2} \Delta_{34}^{2} \Delta_{45}^{2} \Delta_{51}^{2}\right)^{\lambda}} \text {. }
$$

After neglecting the exponential function, and introducing an IR cutoff $R$, the expansion of this integral in Gegenbauer polynomials assumes the form

$$
I^{\prime \prime}=-\frac{\lambda}{2} N_{\lambda}(9,4) \sum_{i, j, k, l, r=0}^{\infty} R_{\lambda}(i, j, k, l, r) A_{\lambda}(i, j, k, l, r)
$$


where $R_{\lambda}(i, j, k, l, r)$ and $A_{\lambda}(i, j, k, l, r)$ denote the corresponding contributions from the radial and angular integrals. They are given by

$$
\begin{aligned}
R_{\lambda}(i, j, k, l, r) & =\int_{0}^{R} \frac{\mathrm{d} r_{1} \mathrm{~d} r_{2} \mathrm{~d} r_{3} \mathrm{~d} r_{4} \mathrm{~d} r_{5} r_{1}^{\lambda} r_{2}^{-\lambda} r_{3}^{\lambda} r_{4}^{-\frac{1}{2}} r_{5}^{-\frac{1}{2}}}{\left(\max _{12} \max _{23} \max _{34} \max _{45} \max _{51}\right)^{\lambda}} \\
A_{\lambda}(i, j, k, l, r) & \left.=\sum_{\substack{m=|l-1| \\
m \neq l}}^{\min _{12}}\right)^{\frac{i}{2}}\left(\frac{\min _{23}}{\max _{23}}\right)^{\frac{j}{2}}\left(\frac{\min _{34}}{\max _{34}}\right)^{\frac{k}{2}}\left(\frac{\min _{45}}{\max _{45}}\right)^{\frac{l}{2}}\left(\frac{\min _{51}}{\max _{51}}\right)^{\frac{r}{2}} \\
& \left.=\delta_{i j} \delta_{i k} \delta_{i m} \delta_{i r} \sum_{\substack{i=|l-1| \\
i \neq l}}^{l+1} \frac{\lambda^{4}}{(i+\lambda)^{4}} D_{\lambda}(1, l, i) \hat{x}_{i} \cdot \hat{x}_{5}\right) C_{r}^{\lambda}\left(\hat{x}_{5} \cdot \hat{x}_{1}\right)
\end{aligned}
$$

Evaluating these expressions, substituting them into (D.12), and expanding the result into a power series with negative powers in $\varepsilon$, we obtain for the pole part after subtraction of the subdivergences

$$
\Longrightarrow=\frac{1}{(4 \pi)^{8}}\left(\frac{1}{6 \varepsilon^{4}}-\frac{1}{6 \varepsilon^{3}}-\frac{3}{4 \varepsilon^{2}}+\frac{1}{\varepsilon}\left(\frac{11}{12}-\zeta(3)\right)\right) \text {, }
$$

where the higher negative powers in $\varepsilon$ indicate that the integral (D.11) contained subdivergences. Substituting the above result into (D.10), we find for the required integral

$$
\gamma_{=}=\frac{1}{(4 \pi)^{8}}\left(\frac{1}{12 \varepsilon^{2}}-\frac{7}{12 \varepsilon}\right) \text {. }
$$

\section{D.2 Sample calculation of $I_{11}$ with four derivatives}

As an example for the computation of the pole part of integrals with four derivatives we show how to obtain $I_{11}$ of Table C.8. This integral contains six propagators which do not end at the root vertex. It is the most complicated one, which we have to compute at four loops. 
Shifting the derivatives to the root vertex, the required integral can be rewritten as

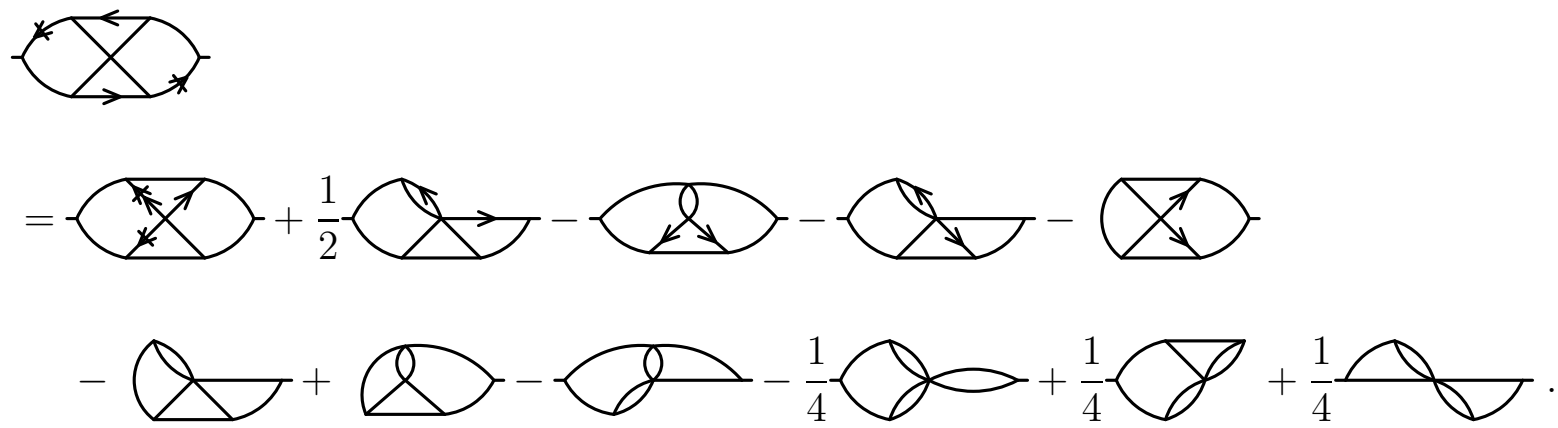

We hence have to compute the pole part of the integral

$$
=\lambda^{3}(1+\lambda) \frac{\Gamma(\lambda)^{10}}{\left(2^{8} \pi^{10}\right)^{1+\lambda}} \int \frac{\mathrm{d}^{D} x_{1} \ldots \mathrm{d}^{D} x_{6} x_{3} \cdot x_{(2} x_{2)} \cdot x_{6} \mathrm{e}^{2 i p \cdot\left(x_{4}-x_{1}\right)}}{x_{2}^{2(\lambda-\alpha+2)}\left(x_{3}^{2} x_{6}^{2}\right)^{1+\lambda}\left(x_{5}^{2} \Delta_{12}^{2} \Delta_{23}^{2} \Delta_{34}^{2} \Delta_{45}^{2} \Delta_{56}^{2} \Delta_{61}^{2}\right)^{\lambda}}
$$

where the parameter $\alpha$ has to be introduced to regularize additional divergences which arise when we neglect the exponential function and introduce the IR cutoff $R$. It should assume the value $\alpha=0$. The parameter $\alpha$ also deforms the (traceless and symmetric) products in the numerator as

$$
x_{3} \cdot x_{(2} x_{2)} \cdot x_{6}=x_{2} \cdot x_{3} x_{2} \cdot x_{6}-\frac{1}{2(1-\alpha+\lambda)} x_{2}^{2} x_{3} \cdot x_{6} .
$$

The expansion of the integral is then given by

$$
\begin{aligned}
I^{\prime \prime \prime \prime}=\frac{\lambda(1+\lambda)}{4} N_{\lambda}(10,4) \sum_{i, j, k, l, r, s=0}^{\infty}( & A_{\lambda}(i, j, k, l, r, s) \\
& \left.-\frac{\lambda}{1-\alpha+\lambda} A_{\lambda}^{\operatorname{tr}}(i, j, k, l, r, s)\right) R_{\lambda}(i, j, k, l, r, s)
\end{aligned}
$$


where the radial and angular integrals are respectively given by

$$
\begin{aligned}
R_{\lambda}(i, j, k, l, r, s) & =\int_{0}^{R} \frac{\mathrm{d} r_{1} \mathrm{~d} r_{2} \mathrm{~d} r_{3} \mathrm{~d} r_{4} \mathrm{~d} r_{5} \mathrm{~d} r_{6} r_{1}^{\lambda} r_{4}^{\lambda} r_{2}^{\alpha-1} r_{3}^{-\frac{1}{2}} r_{6}^{-\frac{1}{2}}}{\left(\max _{12} \max _{23} \max _{34} \max _{45} \max _{56} \max _{61}\right)^{\lambda}} \\
& \left(\frac{\min _{12}}{\max _{12}}\right)^{\frac{i}{2}}\left(\frac{\min _{23}}{\max _{23}}\right)^{\frac{j}{2}}\left(\frac{\min _{34}}{\max _{34}}\right)^{\frac{k}{2}}\left(\frac{\min _{45}}{\max _{45}}\right)^{\frac{l}{2}}\left(\frac{\min _{56}}{\max _{56}}\right)^{\frac{r}{2}}\left(\frac{\min _{61}}{\max _{61}}\right)^{\frac{s}{2}} \\
A_{\lambda}(i, j, k, l, r, s) & =\delta_{i s} \delta_{m k} \delta_{m l} \delta_{m r} \sum_{\substack{m=|i-1| \\
m \neq i}}^{i+1} \sum_{\substack{m=|j-1| \\
m \neq j}}^{j+1} \frac{\lambda^{5}}{(i+\lambda)(m+\lambda)^{4}} D_{\lambda}(1, i, m) D_{\lambda}(1, j, m) C_{m}^{\lambda}(1), \\
A_{\lambda}^{\operatorname{tr}}(i, j, k, l, r, s) & =\delta_{i j} \delta_{i s} \delta_{m k} \delta_{m l} \delta_{m r} \frac{\lambda^{5}}{(i+\lambda)^{2}(m+\lambda)^{3}} \sum_{\substack{m=|i-1| \\
m \neq i}}^{i+1} D_{\lambda}(1, i, m) C_{m}^{\lambda}(1) .
\end{aligned}
$$

Using the identity

$$
\begin{aligned}
& \sum_{m, i, j=0}^{n} \sum_{\substack{m=|i-1| \\
m \neq i}}^{i+1} \sum_{\substack{m=|j-1| \\
m \neq j}}^{j+1} f_{\lambda}(i, j, m) \\
& \quad=f_{\lambda}(1,1,0)+\sum_{m=1}^{n-1} \sum_{a, b= \pm 1} f_{\lambda}(m+a, m+b, m)+\sum_{m=n}^{n+1} f_{\lambda}(m-1, m-1, m)
\end{aligned}
$$

the integral can be cast into the following form

$$
\begin{aligned}
I^{\prime \prime \prime \prime}=\frac{\lambda(1+\lambda)}{4} N_{\lambda}(10,4)( & \Delta f_{\lambda}(1,0)+\sum_{m=1}^{\infty}\left(\Delta f_{\lambda}(m+1, m)+\Delta f_{\lambda}(m-1, m)\right) \\
& \left.+\sum_{m=1}^{\infty}\left(f_{\lambda}(m+1, m-1, m)+f_{\lambda}(m-1, m+1, m)\right)\right)
\end{aligned}
$$

where we have abbreviated

$$
\begin{aligned}
f_{\lambda}(i, j, m) & =\frac{\lambda^{5}}{(i+\lambda)(m+\lambda)^{4}} D_{\lambda}(1, i, m) D_{\lambda}(1, j, m) C_{m}^{\lambda}(1) R_{\lambda}(i, j, m, m, m, i), \\
g_{\lambda}(i, m) & =\frac{\lambda^{6}}{(1-\alpha+\lambda)(i+\lambda)^{2}(m+\lambda)^{3}} D_{\lambda}(1, i, m) C_{m}^{\lambda}(1) R_{\lambda}(i, i, m, m, m, i), \\
\Delta f_{\lambda}(i, m) & =f_{\lambda}(i, i, m)-g_{\lambda}(i, m) .
\end{aligned}
$$


The explicit forms of the functions which appear in the above expressions read

$$
\begin{aligned}
\Delta f_{\lambda}(m-1, m)= & \frac{m((m-1) \lambda-m \alpha) \lambda^{7}}{(1-\alpha+\lambda)(m-1+\lambda)^{3}(m+\lambda)^{4}} C_{m}^{\lambda}(1) \\
& R_{\lambda}(m-1, m-1, m, m, m, m-1) \\
\Delta f_{\lambda}(m+1, m)= & \frac{(m+2 \lambda)(1+m+2 \lambda) \lambda^{8}}{(1+\lambda)(m+1+\lambda)^{3}(m+\lambda)^{4}} C_{m}^{\lambda}(1) \\
& R_{\lambda}(m+1, m+1, m, m, m, m+1), \\
f_{\lambda}(m-1, m+1, m)= & \frac{\lambda^{5}}{(m-1+\lambda)(m+\lambda)^{4}} D_{\lambda}(1, m-1, m) D_{\lambda}(1, m+1, m) C_{m}^{\lambda}(1) \\
& R_{\lambda}(m-1, m+1, m, m, m, m-1) \\
f_{\lambda}(m+1, m-1, m)= & \frac{\lambda^{5}}{(m+1+\lambda)(m+\lambda)^{4}} D_{\lambda}(1, m+1, m) D_{\lambda}(1, m-1, m) C_{m}^{\lambda}(1) \\
& R_{\lambda}(m+1, m-1, m, m, m, m+1) .
\end{aligned}
$$

It is important to remark that one can set $\alpha$ to its correct value $\alpha=0$ in nearly all contributions, except in $\Delta f_{\lambda}(0,1)$, where $m=1$. In this case, some integration domains of the radial integral $R_{\lambda}(0,0,1,1,1,0)$ become divergent as $\frac{1}{\alpha}$ for $\alpha \rightarrow 0$. This divergence is precisely canceled by the prefactor which becomes proportional to $\alpha$ in this case. A finite contribution hence remains, and it has to be considered to obtain the correct answer for the integral. We will comment further on this in a forthcoming publication [39].

Expanding the result into a power series with negative powers in $\varepsilon$, we obtain for the pole part after subtraction of the subdivergences

$$
=\frac{1}{(4 \pi)^{8}}\left(-\frac{1}{32 \varepsilon^{4}}+\frac{1}{12 \varepsilon^{3}}-\frac{19}{96 \varepsilon^{2}}-\frac{1}{\varepsilon}\left(\frac{29}{48}+\frac{5}{2} \zeta(3)-\frac{5}{2} \zeta(5)\right)\right) .
$$

Inserting this result into (D.16), the result for the required integral is hence found to be given by

$$
\nearrow_{-}=\frac{1}{(4 \pi)^{8} \varepsilon}\left(-\frac{1}{4}-\frac{3}{2} \zeta(3)+\frac{5}{2} \zeta(5)\right)
$$




\section{References}

[1] F. Fiamberti, A. Santambrogio, C. Sieg, and D. Zanon, Wrapping at four loops in $\mathcal{N}=4 S Y M$, arXiv:0712.3522

[2] J. M. Maldacena, The large $N$ limit of superconformal field theories and supergravity, Adv. Theor. Math. Phys. 2 (1998) 231-252, hep-th/9711200.

[3] J. A. Minahan and K. Zarembo, The Bethe-ansatz for $\mathcal{N}=4$ super Yang-Mills, JHEP 03 (2003) 013, hep-th/0212208.

[4] N. Beisert, C. Kristjansen, and M. Staudacher, The dilatation operator of $\mathcal{N}=4$ super Yang-Mills theory, Nucl. Phys. B664 (2003) 131-184, hep-th/0303060.

[5] N. Beisert, The su(2|3) dynamic spin chain, Nucl. Phys. B682 (2004) 487-520, hep-th/0310252.

[6] M. Staudacher, The factorized S-matrix of CFT/AdS, JHEP 05 (2005) 054, hep-th/0412188.

[7] N. Beisert, V. Dippel, and M. Staudacher, A novel long range spin chain and planar $\mathcal{N}=4$ super Yang-Mills, JHEP 07 (2004) 075, hep-th/0405001.

[8] C. Sieg and A. Torrielli, Wrapping interactions and the genus expansion of the 2- point function of composite operators, Nucl. Phys. B723 (2005) 3-32, hep-th/0505071].

[9] T. Fischbacher, T. Klose, and J. Plefka, Planar plane-wave matrix theory at the four loop order: Integrability without BMN scaling, JHEP 02 (2005) 039, hep-th/0412331.

[10] J. Ambjorn, R. A. Janik, and C. Kristjansen, Wrapping interactions and a new source of corrections to the spin-chain / string duality, Nucl. Phys. B736 (2006) 288-301, hep-th/0510171.

[11] R. A. Janik and T. Lukowski, Wrapping interactions at strong coupling - the giant magnon, Phys. Rev. D76 (2007) 126008, arXiv:0708.2208 [hep-th]].

[12] A. Rej, D. Serban, and M. Staudacher, Planar $\mathcal{N}=4$ gauge theory and the Hubbard model, JHEP 03 (2006) 018, hep-th/0512077.

[13] A. V. Kotikov, L. N. Lipatov, A. Rej, M. Staudacher, and V. N. Velizhanin, Dressing and Wrapping, J. Stat. Mech. 0710 (2007) P10003, arXiv:0704.3586 [hep-th].

[14] L. N. Lipatov, Reggeization of the Vector Meson and the Vacuum Singularity in Nonabelian Gauge Theories, Sov. J. Nucl. Phys. 23 (1976) 338-345.

[15] E. A. Kuraev, L. N. Lipatov, and V. S. Fadin, The Pomeranchuk Singularity in Nonabelian Gauge Theories, Sov. Phys. JETP 45 (1977) 199-204.

[16] I. I. Balitsky and L. N. Lipatov, The Pomeranchuk Singularity in Quantum Chromodynamics, Sov. J. Nucl. Phys. 28 (1978) 822-829.

[17] J. Penedones and P. Vieira, Toy models for wrapping effects, arXiv:0806.1047.

[18] G. Arutyunov, S. Frolov, and M. Zamaklar, Finite-size effects from giant magnons, Nucl. Phys. B778 (2007) 1-35, hep-th/0606126.

[19] S. Schafer-Nameki, M. Zamaklar, and K. Zarembo, How accurate is the quantum string Bethe ansatz?, JHEP 12 (2006) 020, hep-th/0610250. 
[20] S. Schafer-Nameki, Exact expressions for quantum corrections to spinning strings, Phys. Lett. B639 (2006) 571-578, hep-th/0602214.

[21] N. Gromov, S. Schafer-Nameki, and P. Vieira, Quantum Wrapped Giant Magnon, arXiv:0801.3671.

[22] J. A. Minahan and O. Ohlsson Sax, Finite size effects for giant magnons on physical strings, arXiv:0801.2064

[23] M. P. Heller, R. A. Janik, and T. Lukowski, A new derivation of Luscher F-term and fluctuations around the giant magnon, arXiv:0801.4463,

[24] B. Ramadanovic and G. W. Semenoff, Finite Size Giant Magnon, arXiv:0803.4028.

[25] Y. Hatsuda and R. Suzuki, Finite-Size Effects for Dyonic Giant Magnons, Nucl. Phys. B800 (2008) 349-383, [arXiv:0801.0747].

[26] K. G. Chetyrkin, A. L. Kataev, and F. V. Tkachov, New Approach to Evaluation of Multiloop Feynman Integrals: The Gegenbauer Polynomial x Space Technique, Nucl. Phys. B174 (1980) $345-377$.

[27] D. I. Kazakov, Calculation of Feynman integrals by the method of 'uniqueness', Theor. Math. Phys. 58 (1984) 223-230.

[28] N. Beisert, B. Eden, and M. Staudacher, Transcendentality and crossing, J. Stat. Mech. 0701 (2007) P021, hep-th/0610251.

[29] C. A. Keeler and N. Mann, Wrapping Interactions and the Konishi Operator, arXiv:0801.1661.

[30] S. J. Gates, M. T. Grisaru, M. Rocek, and W. Siegel, Superspace, or one thousand and one lessons in supersymmetry, Front. Phys. 58 (1983) 1-548, hep-th/0108200.

[31] N. Beisert, T. McLoughlin, and R. Roiban, The Four-Loop Dressing Phase of $\mathcal{N}=4$ SYM, Phys. Rev. D76 (2007) 046002, arXiv:0705.0321 [hep-th].

[32] G. Arutyunov, S. Frolov, and M. Staudacher, Bethe ansatz for quantum strings, JHEP 10 (2004) 016, hep-th/0406256.

[33] R. Hernandez and E. Lopez, Quantum corrections to the string Bethe ansatz, JHEP 07 (2006) 004, hep-th/0603204.

[34] N. Beisert, R. Hernandez, and E. Lopez, A crossing-symmetric phase for $A d S_{5} \times S^{5}$ strings, JHEP 11 (2006) 070, hep-th/0609044.

[35] N. Beisert and T. Klose, Long-range gl(n) integrable spin chains and plane-wave matrix theory, J. Stat. Mech. 0607 (2006) P006, hep-th/0510124.

[36] Z. Bajnok and R. A. Janik, Four-loop perturbative Konishi from strings and finite size effects for multiparticle states, arXiv:0807.0399.

[37] S. A. Larin, F. V. Tkachov, and J. A. M. Vermaseren, The FORM version of MINCER, NIKHEF-H/91-18.

[38] W. Celmaster and R. J. Gonsalves, Fourth Order QCD Contributions to the e+ e-Annihilation Cross-Section, Phys. Rev. D21 (1980) 3112.

[39] F. Fiamberti, A. Santambrogio, and C. Sieg, to appear. 